Mon. Not. R. Astron. Soc. 000, 1-?? (0000) Printed 15 September $2018 \quad$ (MN LATEX style file v2.2)

\title{
Constraints on warm dark matter from weak lensing in anomalous quadruple lenses
}

\author{
Kaiki Taro Inoue ${ }^{1 \star}$, Ryuichi Takahashi $^{2}$, Tomo Takahashi $^{3}$ and Tomoaki Ishiyama ${ }^{4}$ \\ ${ }^{1}$ Department of Science and Engineering, Kinki University, Higashi-Osaka, Osaka, 577-8502, Japan \\ ${ }^{2}$ Faculty of Science and Technology, Hirosaki University, 3 Bunkyo-cho, Hirosaki, Aomori 036-8561, Japan \\ ${ }^{3}$ Department of Physics, Saga University, Saga 840-8502, Japan \\ ${ }^{4}$ Center for Computational Science, University of Tsukuba, 1-1-1, Tennodai, Tsukuba, Ibaraki 305-8577, Japan
}

15 September 2018

\begin{abstract}
We investigate the weak lensing effect by line-of-sight structures with a surface mass density of $\lesssim 10^{8} M_{\odot} / \operatorname{arcsec}^{2}$ in QSO-galaxy quadruple lens systems. Using highresolution $N$-body simulations in warm dark matter (WDM) models and observed four quadruple lenses that show anomalies in the flux ratios, we obtain constraints on the mass of thermal WDM, $m_{\mathrm{WDM}} \geqslant 1.3 \mathrm{keV}(95 \% \mathrm{CL})$ assuming that the density of the primary lens is described by a singular isothermal ellipsoid (SIE). The obtained constraint is consistent with those from Lyman- $\alpha$ forests and the number counts of high-redshift galaxies at $z>4$. Our results show that WDM with a free-streaming comoving wavenumber $k_{\mathrm{fs}} \leqslant 27 \mathrm{~h} / \mathrm{Mpc}$ is disfavoured as the major component of cosmological density at redshifts $0.5 \lesssim z \lesssim 4$ provided that the SIE models describe the gravitational potentials of the primary lenses correctly.
\end{abstract}

Key words: galaxies: formation - cosmology: theory - dark matter

\section{INTRODUCTION}

The clustering property of dark haloes at spatial scales of $\lesssim 1 \mathrm{Mpc}$ is far from being understood. In particular, the number of satellite galaxies in our Galaxy is by far smaller " than expected from theory, which is the so-called "missing satellite problem." As a solution, we may consider: (1) baryonic solution - the star formation in the satellite galaxy is suppressed due to some baryonic process. (2) dark matter solution - a number of satellite galaxies are suppressed due to a large free-streaming scale of dark matter particles.

It has been known that the flux ratios of lensed images in some quadruply lensed QSOs disagree with the prediction of best-fitting lens models with a smooth potential whose fluctuation scale is larger than the separation between the lensed images. Such a discrepancy is called the 'anomalous flux ratio' and has been considered as an imprint of cold dark matter $(\mathrm{CDM})$ subhaloes with a mass of $\sim 10^{8-9} M_{\odot}$ in the lensing galaxy (Mao \& Schneider 1998; Metcalf \& Madau 2001; Chiba 2002; Dalal \& Kochanek 2002; Metcalf et al. 2004; Chiba et al. 2005; Sugai et al. 2007; McKean et al. 2007; More et al. 2009; Minezaki et al. 2009).

However, recent studies based on high resolution simulations suggested that the predicted substructure population is too low to explain the observed anomalous flux ratios

\footnotetext{
* E-mail:kinoue@phys.kindai.ac.jp
}

(Amara et al. 2006; Maccio \& Miranda 2006; Chen 2009; Xu et al. 2009, 2010; Chen et al. 2011). Moreover, the formation of dark satellites in lensing galaxies can be suppressed by baryonic processes, such as tidal stripping and outflows due to supernovae. If the number density of satellites in our Galaxy represents a typical value, the surface mass of dark satellites in lensing galaxies should also be smaller than the expected values obtained from $N$-body simulations.

Intergalactic haloes that are not belonging to lensing galaxies may evade a suppression due to baryonic processes. Moreover, the lensing effects due to line-of-sight haloes may play an important role (Chen et al. 2003; Metcalf 2005; Xu et al. 2012). Intergalactic non-linear structures such as voids, walls, and filaments could also influence the flux ratios significantly.

Indeed, taking into account astrometric shifts, recent studies have found that the observed anomalous flux ratios can be explained solely by these line-of-sight structures with surface mass density $\sim 10^{7-8} M_{\odot} / \operatorname{arcsec}^{2}$ (Inoue \& Takahashi 2012; Takahashi \& Inoue 2014) without dark subhaloes in the lensing galaxies taken into account. The observed increase in the amplitude of magnification perturbations with redshift strongly implies that the origin is associated with sources rather than lenses. If this is the case, one does not need to care about the suppression of dark satellites in the lensing galaxy due to baryonic processes as a number of 


\section{Kaiki Taro Inoue, Ryuichi Takahashi, Tomo Takahashi, Tomoaki Ishiyama}

minihaloes in the line of sight are not belonging to massive galaxies.

Another mechanism that can suppress the number of dwarf galaxies is the free-streaming of dark matter particles. If the thermal velocity at the decoupling from the thermal bath is large enough, dark matter particles would erase the primordial fluctuations at scales of dwarf galaxies. Warm dark matter (WDM) particles are candidates for achieving such suppression.

However, if the suppression is too strong, the amount of neutral hydrogen such as Lyman- $\alpha$ clouds is also significantly reduced. In fact, the best constraint on the mass scale of WDM comes from the observations of Lyman- $\alpha$ forests (Viel et al. 2005; Seljak et al. 2006; Boyarsky et al. 2009; Viel et al. 2013).

In a similar manner, one can constrain the mass or the free-streaming scale of dark matter particles using anomalous quadruple lenses (Miranda \& Maccio 2007). If the freestreaming scale is too large, or equivalently, the particle mass is too small, the amplitude of fluctuations of a surface mass density in the line of sight becomes so small that the weak gravitational lensing effect, which acts as a perturbation to the flux ratios, becomes negligible. Therefore, the observed anomalous flux ratios cannot be explained in such dark matter models.

In this paper, we revisit the weak lensing effect by the line-of-sight structures in WDM models taking into account two important non-linear effects that have been overlooked in the literature. One is the quick regeneration of the suppressed power of WDM models and the catching up with the linear and non-linear power of the CDM models (Boehm et al. 2005; Schneider et al. 2012). This effect might make WDM models difficult to exclude using QSO-galaxy lensing systems. Another is the weak lensing effect due to non-linear objects such as walls, voids, and filaments. In CDM models, it turned out that the weak lensing effect from locally underdense regions is also important for estimating magnification perturbation by the line-of-sight structures (Takahashi \& Inoue 2014). The weak lensing effect due to walls and filaments could also be important. Therefore, we need to incorporate lensing effects due to non-linear objects in WDM models as well. For simplicity, however, we do not consider lensing effects due to subhaloes in the lensing galaxies.

To take into account such non-linear effects, we first calculate the non-linear power spectra of matter fluctuations down to mass scales of $\sim 10^{5} h^{-1} M_{\odot}$ using $N$-body simulations. For simplicity, we do not consider baryonic effects in our simulations. Then we estimate the probability distribution of magnification perturbation for each lens using the semi-analytic formulae developed in Takahashi \& Inoue (2014). We also take into account the astrometric shifts due to line-of-sight structures, which are often overlooked in the literature.

In Section 2, we describe our semi-analytic formulation for calculating the magnification perturbation due to lineof-sight structures. In Section 3, we show the results of our $N$-body simulations and the obtained non-linear power spectra in WDM models. In Section 4, we describe our samples of QSO-galaxy lensing systems that show anomalies in the flux ratios. In Section 5, we present our results on the constraints on the mass of WDM particles and the free-streaming scales

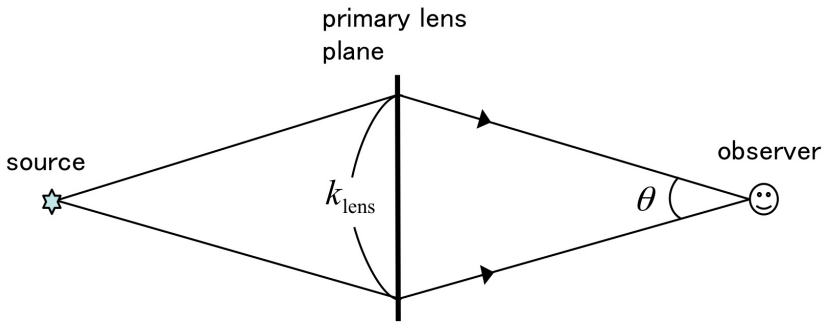

Figure 1. Schematic diagram of unperturbed light rays. The wavenumber $k_{\text {lens }}$ is defined as $k_{\text {lens }}=\pi / 2 b$, where $b$ is the mean separation between lensed images and the centroid of the primary lens galaxy.

of dark matter particles. In Section 6, we conclude and discuss some relevant issues.

In what follows, we assume a cosmology with a current matter density $\Omega_{m, 0}=0.3134$, a baryon density $\Omega_{b, 0}=0.0487$, a cosmological constant $\Omega_{\Lambda, 0}=0.6866$, a Hubble constant $H_{0}=67.3 \mathrm{~km} / \mathrm{s} / \mathrm{Mpc}$, a spectral index $n_{s}=0.9603$, and the root-mean-square (rms) amplitude of matter fluctuations at $8 h^{-1} \mathrm{Mpc}, \sigma_{8}=0.8421$, which are obtained from the observed cosmic microwave background (Planck + WMAP polarization, Planck Collaboration et al. (2014)).

\section{SEMI-ANALYTIC FORMULATION}

In this section, we briefly describe our semi-analytical formulation (for details, see Inoue \& Takahashi (2012); Takahashi \& Inoue (2014)).

We use a statistic $\eta$ to measure the magnification perturbation of lensed images in QSO-galaxy lens systems:

$$
\eta \equiv\left[\frac{1}{2 N_{\text {pair }}} \sum_{\mathrm{i} \neq \mathrm{j}}\left[\delta_{\mathrm{i}}^{\mu}(\text { minimum })-\delta_{\mathrm{j}}^{\mu}(\text { saddle })\right]^{2}\right]^{1 / 2}
$$

where $\delta^{\mu}$ (minimum) and $\delta^{\mu}$ (saddle) are magnification (denoted by $\mu$ ) contrasts $\delta^{\mu} \equiv \delta \mu / \mu$ corresponding to the minimum and saddle images and $N_{\text {pair }}$ denotes the number of pairs of lensed images. If the correlation of magnification between pairs of images is negligible, then $\eta$ corresponds to the mean magnification perturbation of one of lensed images. For instance, $\eta=0.1$ means that the magnification is expected to change by 10 percent. Note that we need to fix the primary lens model (i.e., a best-fittingted model without line-of-sight structures) in order to calculate $\eta$. In other words, $\eta$ is a model dependent statistic.

The second moment of the magnification perturbation $\eta$ can be calculated as follows. First, we need to estimate a perturbation $\varepsilon$ to the largest angular separation $\theta_{\max }$ between a pair of lensed images $\mathrm{X}$ and $\mathrm{Y}$ due to the line-of-sight structures,

$$
\varepsilon=|\delta \boldsymbol{\theta}(\mathrm{X})-\delta \boldsymbol{\theta}(\mathrm{Y})|,
$$

where $\delta \boldsymbol{\theta}$ represents the astrometric shift perturbation of a lensed image at $\boldsymbol{\theta}$ in the lens plane. We then assume that the perturbation satisfies $\varepsilon \leqslant \varepsilon_{0}$, where $\varepsilon_{0}$ is the observational error for the largest angular separation. This condition gives 
an approximated upper limit on the contribution of line-ofsight structures assuming that the gravitational potential of the primary lens is sufficiently smooth on the scale of the Einstein radius and the projected density has a nearlycircular elliptical symmetry (see appendix in Takahashi \& Inoue (2014)).

In order to satisfy such a condition, we assume that small-scale modes with a wavelength larger than the mean comoving separation $b$ between the lens centre and lensed images at the primary lens plane are significantly suppressed. Any modes whose fluctuation scales are larger than $b$, which is roughly the size of the comoving Einstein radius, contribute to the smooth component of a primary lens, namely, a constant convergence and shear (Fig. 1). Therefore, we consider only modes whose wavenumbers satisfy $k>k_{\text {lens }}$ where $k_{\text {lens }} \equiv \pi / 2 b$. Otherwise, double-counting of the constant convergence and shear leads to a systematically large perturbation. Furthermore, we also assume that modes with wavenumbers $k_{\text {lens }}<k<k_{\text {cut }}$ are suppressed to some extent. These modes correspond to the secondary lens [modelled by a singular isothermal sphere (SIS) or singular isothermal ellipsoid (SIE)] in the line of sight. In our samples, MG0414+0534, B1608+656 and B2045+265 have the secondary lens. In our sample, the lensing galaxies of these systems $\left(k_{\text {lens }}=100 \sim 200 h / \mathrm{Mpc}\right)$ are typically more massive than those without a secondary lens $\left(k_{\text {lens }}=400 \sim 500 h / \mathrm{Mpc}\right)$. The cut off scale $k_{\text {cut }}$ is determined by the condition that the perturbation $\varepsilon$ of an angular separation $\theta$ between an arbitrary pair of lensed images should not exceed the observational error $\varepsilon_{\text {obs }}$ for the maximum separation angle between lensed images.

In what follows, we adopt a filtering the so-called constant shift (CS) filter (Takahashi \& Inoue 2014),

$$
W_{\mathrm{CS}}\left(k ; k_{\text {cut }}\right)= \begin{cases}W_{\text {int }}(k), & k<k_{\text {cut }} \\ 1, & k \geqslant k_{\text {cut }}\end{cases}
$$

in which the corresponding contribution to the angular shifts between a pair of images with the maximum separation angle $\theta_{\max }$ is constant in logarithmic interval in $k$ for $k<k_{\text {cut }}$. The CS filter mildly suppresses the large angularscale modes with wavenumbers $k_{\text {lens }}<k<k_{\text {cut }}$ by keeping the contribution to an angular shift $\varepsilon$ constant in $\log k$ and gives a relatively good approximation in the CDM models (Takahashi \& Inoue 2014).

$W_{\text {int }}$ is explicitly given by

$$
W_{\text {int }}^{2}\left(k ; k_{\text {cut }}\right) \equiv \frac{\left.\frac{\partial\left\langle\varepsilon^{2}\right\rangle}{\partial \ln k}\right|_{k=k_{\text {cut }}}}{\frac{\partial\left\langle\varepsilon^{2}\right\rangle}{\partial \ln k}}
$$

where

$$
\left\langle\varepsilon^{2}\right\rangle=2\left\langle\delta \theta^{2}(0)\right\rangle-2\left\langle\delta \theta(0) \delta \theta\left(\theta_{\max }\right)\right\rangle
$$

and

$$
\begin{aligned}
\langle\delta \theta(0) \delta \theta(\theta)\rangle & =\frac{9 H_{0}^{4} \Omega_{m, 0}^{2}}{c^{4}} \int_{0}^{r_{S}} d r\left(\frac{r-r_{S}}{r_{S}}\right)^{2}[1+z(r)]^{2} \\
& \times \int_{k_{\text {lens }}}^{\infty} \frac{d k}{2 \pi k} W_{\mathrm{CS}}^{2}\left(k ; k_{\text {cut }}\right) P_{\delta}(k, r) J_{0}(g(r) k \theta)
\end{aligned}
$$

where

$$
g(r)= \begin{cases}r, & r<r_{L} \\ r_{L}\left(r_{S}-r\right) /\left(r_{S}-r_{L}\right), & r \geqslant r_{L}\end{cases}
$$

and $P_{\delta}(k, r)$ is the power spectrum of dark matter density fluctuations as a function of the wavenumber $k$ and the comoving distance $r . r_{S}$ is the comoving distance to the source and $r_{L}$ to the lens from an observer and $z(r)$ is the redshift of a point at a comoving distance $r$. \langle\rangle represents an ensemble average. $J_{0}$ is the zero-th order Bessel function. $g(r) \theta$ denotes the tangential separation between two unperturbed light rays at a comoving distance $r$ from the observer.

Once $k_{\text {lens }}$ and $k_{\text {cut }}$ are determined, we can compute the constrained perturbed convergence $\delta \kappa$ and shear $\delta \gamma_{1,2}$ as functions of a separation angle $\boldsymbol{\theta}$ between a pair of lensed images. For instance, the constrained two-point correlation of $\delta \kappa$ as a function of a separation angle $\boldsymbol{\theta}$ is

$$
\begin{aligned}
\xi_{\kappa \kappa}(\boldsymbol{\theta}) \equiv & \langle\delta \kappa(0) \delta \kappa(\boldsymbol{\theta})\rangle \\
= & \frac{9 H_{0}^{4} \Omega_{m, 0}^{2}}{4 c^{4}} \int_{0}^{r_{S}} d r r^{2}\left(\frac{r-r_{S}}{r_{S}}\right)^{2}[1+z(r)]^{2} \\
& \times \int_{k_{\text {lens }}}^{\infty} \frac{d k}{2 \pi} k W_{\mathrm{CS}}^{2}\left(k ; k_{\text {cut }}\right) P_{\delta}(k, r) J_{0}(g(r) k \theta) .
\end{aligned}
$$

To calculate $P_{\delta}$, we use a fitting function obtained from high resolution cosmological simulations (see also Smith et al. (2003); Inoue \& Takahashi (2012); Takahashi et al. (2012); Takahashi \& Inoue (2014)). The fitting function for the WDM model can be used up to a wavenumber $k \sim 300 \mathrm{hMpc}^{-1}$ at $0 \leqslant z \leqslant 3$ within $\sim 20 \%$ accuracy (see Section 3).

The two-point correlation functions for the other perturbed quantities are obtained by the following substitution in equation (8):

$$
\begin{array}{rll}
\left\langle\delta \gamma_{1}(0) \delta \gamma_{1}(\boldsymbol{\theta})\right\rangle & : & J_{0} \rightarrow \frac{1}{2}\left[J_{0}+J_{4} \cos \left(4 \phi_{\theta}\right)\right], \\
\left\langle\delta \gamma_{2}(0) \delta \gamma_{2}(\boldsymbol{\theta})\right\rangle & : & J_{0} \rightarrow \frac{1}{2}\left[J_{0}-J_{4} \cos \left(4 \phi_{\theta}\right)\right], \\
\left\langle\delta \kappa(0) \delta \gamma_{1}(\boldsymbol{\theta})\right\rangle: & J_{0} \rightarrow-J_{2} \cos \left(2 \phi_{\theta}\right), \\
\left\langle\delta \kappa(0) \delta \gamma_{2}(\boldsymbol{\theta})\right\rangle & : & J_{0} \rightarrow-J_{2} \sin \left(2 \phi_{\theta}\right), \\
\left\langle\delta \gamma_{1}(0) \delta \gamma_{2}(\boldsymbol{\theta})\right\rangle & : & J_{0} \rightarrow \frac{1}{2} J_{4} \sin \left(4 \phi_{\theta}\right),
\end{array}
$$

where $\boldsymbol{\theta}=\left(\theta \cos \phi_{\theta}, \theta \sin \phi_{\theta}\right)$ and the Bessel functions $J_{0,2,4}$ are functions of $g(r) k \theta$. From equations (1), (8) and (9), we can obtain the second moment of $\eta$.

For example, let us consider three images with two minima $\mathrm{A}$ and $\mathrm{C}$ and one saddle $\mathrm{B}$ with $\kappa_{B}<1$. Choosing coordinates where the separation angle is perpendicular to + mode (i.e., $\theta \sin \phi_{\theta}=0$ ), we have $\left\langle\delta \kappa \delta \gamma_{2}\right\rangle=\left\langle\delta \gamma_{1} \delta \gamma_{2}\right\rangle=0$. Then, for $\left|\delta_{i}^{\mu}\right| \ll 1$, the second moment $\left\langle\eta^{2}\right\rangle$ can be written as

$$
\begin{aligned}
\left\langle\eta^{2}\right\rangle & =\frac{1}{4}\left[\left(I_{A}+I_{B}\right)-2 I_{A B}\left(\theta_{A B}\right)+\left(I_{B}+I_{C}\right)\right. \\
& \left.-2 I_{B C}\left(\theta_{B C}\right)\right],
\end{aligned}
$$

where

$$
I_{i} \equiv \mu_{i}^{2}\left(4\left(1-\kappa_{i}\right)^{2}+2 \gamma_{1 i}^{2}+2 \gamma_{2 i}^{2}\right) \xi_{\kappa}(0)
$$


and

$$
\begin{aligned}
I_{i j}(\theta) & \equiv 4 \mu_{i} \mu_{j}\left[\left(1-\kappa_{i}\right)\left(1-\kappa_{j}\right) \xi_{\kappa}(\theta)\right. \\
& +\gamma_{1 i} \gamma_{1 j}\left\langle\delta \gamma_{1}(0) \delta \gamma_{1}(\theta)\right\rangle+\gamma_{2 i} \gamma_{2 j}\left\langle\delta \gamma_{2}(0) \delta \gamma_{2}(\theta)\right\rangle \\
& +\left(1-\kappa_{i}\right) \gamma_{1 j}\left\langle\delta \kappa_{i}(0) \delta \gamma_{1 j}(\theta)\right\rangle \\
& \left.+\left(1-\kappa_{j}\right) \gamma_{1 i}\left\langle\delta \kappa_{j}(0) \delta \gamma_{1 i}(\theta)\right\rangle\right]
\end{aligned}
$$

for $i=\mathrm{A}, \mathrm{B}, \mathrm{C}$. In a similar manner, for a four-image system with two minima $\mathrm{A}$ and $\mathrm{C}$ and two saddles $\mathrm{B}$ and $\mathrm{D}$ with $\kappa_{\mathrm{B}}<1$ and $\kappa_{\mathrm{D}}<1$, the second moment is given by

$$
\begin{aligned}
\left\langle\eta^{2}\right\rangle & =\frac{1}{8}\left[I_{A}+I_{B}-2 I_{A B}\left(\theta_{A B}\right)+\left(I_{C}+I_{B}\right)\right. \\
& -2 I_{C B}\left(\theta_{C B}\right)+\left(I_{A}+I_{D}\right)-2 I_{A D}\left(\theta_{A D}\right) \\
& \left.+\left(I_{C}+I_{D}\right)-2 I_{C D}\left(\theta_{C D}\right)\right]
\end{aligned}
$$

where $I_{i}$ and $I_{i j}(\theta), i=\mathrm{A}, \mathrm{B}, \mathrm{C}, \mathrm{D}$ are given by equations (11) and (12). Note that we are using coordinates in which $\phi_{\theta}=0$.

\section{NON-LINEAR POWER SPECTRUM}

\subsection{Initial condition}

We calculate the initial power spectrum in models with WDM by using the modified version of CAMB (Lewis et al. 2000). We assume thermal distribution for WDM and all dark matter component being WDM. Since we fix the abundance of WDM, its mass $m_{\mathrm{WDM}}$ and the temperature of WDM species $T_{\mathrm{WDM}}$ are related as

$$
\Omega_{\mathrm{WDM}} h^{2}=\left(\frac{T_{\mathrm{WDM}}}{T_{\nu}}\right)^{3}\left(\frac{m_{\mathrm{WDM}}}{94 \mathrm{eV}}\right),
$$

where $T_{\nu}$ is the temperature of neutrinos. By the effect of the free-streaming of WDM particles, the cosmic structure can be erased and the matter power spectrum damps on small scales, which is commonly characterized by the freestreaming scale $\lambda_{\mathrm{fs}}$, defined by the comoving length that WDM particles free-stream until the radiation-matter equality time. $\lambda_{\mathrm{fs}}$ is explicitly given by (Kolb \& Turner 1990)

$$
\begin{aligned}
\lambda_{\mathrm{fs}} & =0.114 \mathrm{Mpc}\left(\frac{1 \mathrm{keV}}{m_{\mathrm{WDM}}}\right)\left(\frac{10.75}{g_{*}\left(T_{D}\right)}\right)^{1 / 3} \\
& \times\left[2+\log \left(\frac{t_{\mathrm{eq}}}{t_{\mathrm{NR}}}\right)\right],
\end{aligned}
$$

where $t_{\mathrm{eq}}$ and $t_{\mathrm{NR}}$ are the time of radiation-matter equality and that when WDM particles become non-relativistic, respectively. $g_{*}\left(T_{D}\right)$ is the effective number of degrees of freedom at the time of decoupling of WDM particles, denoted by the temperature $T=T_{D}$. In the following analysis, we fix the energy density of WDM as $\Omega_{\mathrm{WDM}}=0.2647$; hence, the temperature $T_{\mathrm{WDM}}\left(\right.$ or $g_{*}\left(T_{D}\right)$ ) and the mass $m_{\mathrm{WDM}}$ are related through equation (14).

Above arguments are valid for thermally produced WDM species. However, other candidates for WDM such as sterile neutrinos (Dodelson \& Widrow 1994) have also been

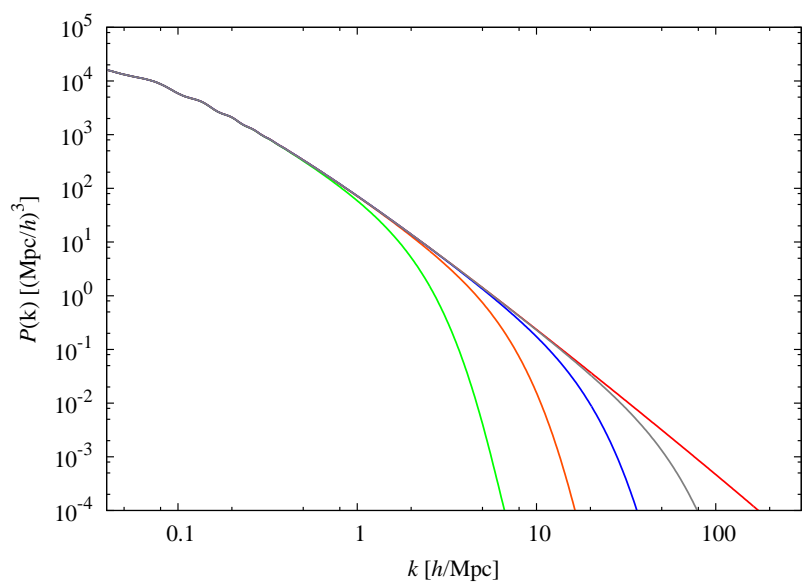

Figure 2. Plots of linear matter power spectra for the $\Lambda$ CDM model (red solid line), WDM models with $k_{\mathrm{fs}}=2 \pi / \lambda_{\mathrm{fs}}=$ $140 h / \mathrm{Mpc}$ (grey), $44 h / \mathrm{Mpc}$ (blue), $15 h / \mathrm{Mpc}$ (orange) and $4.8 \mathrm{~h} / \mathrm{Mpc}$ (green).

discussed in the literature. For the sterile neutrinos produced via active-sterile neutrino oscillations, its distribution function can be approximated by a generalized Fermi-Dirac distribution, then the effect of sterile neutrino can be regarded as the same as the one for WDM by using the following identification for the mass (Colombi et al. 1996; Viel et al. 2005):

$$
m_{\mathrm{s}}=4.46 \mathrm{keV}\left(\frac{m_{\mathrm{WDM}}}{1 \mathrm{keV}}\right)^{4 / 3}\left(\frac{0.12}{\Omega_{\mathrm{WDM}} h^{2}}\right)^{1 / 3} .
$$

From this formula, one can derive the constraint for the mass of sterile neutrino once we obtain that for thermally produced WDM.

In Fig. 2, we show the linear matter power spectra in the $\Lambda$ CDM model, and WDM models with $k_{\mathrm{fs}}=2 \pi / \lambda_{\mathrm{fs}}=$ $140,44,15$ and $4.8 \mathrm{~h} / \mathrm{Mpc}$. The corresponding WDM masses are listed in Table 1 .

\subsection{N-body simulation}

We run cosmological $N$-body simulations to investigate the non-linear matter power spectra of WDM models. Our purpose is to obtain the fitting formula of non-linear power spectra used in our analytical formula (see Section 2). In order to cover a wide-range scale of gravitational evolution, we run simulations with two different boxes with a side of $100 h^{-1} \mathrm{Mpc}$ and $10 h^{-1} \mathrm{Mpc}$, hereinafter referred to as $L 100$ and $L 10$, respectively. The number of particles in the boxes is set to $1024^{3}$. The initial positions and velocities of particles are given at redshift $z_{\text {init }}=24$ based on second-order Lagrangian perturbation theory (Crocce et al. 2006; Nishimichi et al. 2009). We adopt a concordant CDM model and four WDM models with free-streaming wavenumbers $k_{\mathrm{fs}}=2 \pi / \lambda_{\mathrm{fs}}=140,44,15$ and $4.8 \mathrm{~h} / \mathrm{Mpc}$ in our simulations. The CDM and WDM models are summarized in Table 1. The input linear power spectra of the CDM and WDM models are evaluated using CAMB (see Section 3.1). In our simulations, we ignore the thermal motion of WDM particles, which can be verified as follows. The rms thermal velocity of WDM particles at the initial redshift $\left(z_{\text {init }}=24\right)$ is 
$\sigma_{v} \simeq 1.1 \mathrm{~km} / \mathrm{s}\left(g_{\mathrm{WDM}} / 1.5\right)^{1 / 3}\left(m_{\mathrm{WDM}} / \mathrm{keV}\right)^{-4 / 3}$ in our cosmological model, where $g_{\mathrm{WDM}}$ is the degree of freedom of the WDM particle (Bode et al. 2001). On the other hand, the rms physical peculiar velocity of the particles at the initial time is $\gtrsim 10 \mathrm{~km} / \mathrm{s}$ in our WDM models. Thus, we can ignore the thermal motion of WDM particles (see also similar discussion in Angulo et al. (2013)).

To follow the gravitational evolution of the dark matter particles, we employ publicly available tree-PM codes, Gadget2 (Springel et al. 2001; Springel 2005) for the large-box simulation (L100) and GreeM (Ishiyama et al. 2009, 2012) for the small-box simulation (L10). GreeM is tuned to accelerate the tree gravitational calculation, and it is faster than Gadget2 especially in the strongly non-linear regime. Hence, we employ GreeM for the small-box simulation. The PM meshes are $2048^{3}\left(512^{3}\right)$ for the L100 (L10). The particle Nyquist wavenumbers are $k_{\mathrm{Nyq}}=32.2(322) h / \mathrm{Mpc}$ for the L100 (L10). The gravitational softening length is set to $3 \%$ of the mean particle separation. The simulation snapshots are dumped at redshifts $z=0,0.3,0.6,1,2$ and 3 . We prepare 3(5) independent realizations for the L100 (L10) for each CDM or WDM model to reduce the sample variance. Our simulation settings are summarized in Table 2.

We check the accuracy of our simulation results as follows. For Gadget2, we use the same simulation parameters (time step, force accuracy and so on) in Takahashi et al. (2012) (Section 2) in which we achieved a few percent accuracy of the power spectra. For GreeM, we run simulations with finer simulation parameters and confirmed that the power spectra have $<1 \%$ accuracy for $k<300 h / \mathrm{Mpc}$.

To evaluate the matter power spectra from the particle distribution, we assign $1024^{3}$ particles into $1536^{3}$ grids using the Cloud-in-Cell (CIC) method (Hockney \& Eastwood 1988) to obtain the density fluctuations. Then, we perform $\mathrm{FFT}^{1}$ and calculate the power spectrum:

$$
P(k)=\frac{1}{N_{k}} \sum_{\boldsymbol{k}^{\prime}}\left|\tilde{\delta}\left(\boldsymbol{k}^{\prime}\right)\right|^{2},
$$

where the summation is done over a range of $k-\Delta k / 2<$ $\left|\boldsymbol{k}^{\prime}\right|<k+\Delta k / 2$ with a bin-width $\Delta k$, and $N_{k}$ is the number of modes in a $k$ bin. We also employ the holding method (e.g. Jenkins et al. 1998; Smith et al. 2003) to probe smaller scales. We calculate the mean power spectra and $1 \sigma$ errors from 5(3) realizations in the L100(L10).

Fig. 3 shows our simulation results for the matter power spectra in the CDM and WDM models shown in Table 1 at redshifts $z=0,0.3,1$ and 2 . The filled circles with error bars are the mean power spectra with the errors obtained from the realizations of simulations. The results are taken from the large-box simulations (L100) for $k<30 h / \mathrm{Mpc}$ and from the small-box simulations (L10) for $k>60 h / \mathrm{Mpc}$. Here, $k=30 h / \mathrm{Mpc}$ is the Nyquist wavenumber of the L100, and $k=60 h / \mathrm{Mpc}$ corresponds to a scale of $1 / 10$ times smaller than the small box-size $(\mathrm{L} 10)^{2}$. The vertical dotted line denotes the Nyquist wavenumber of the small-box

1 FFTW home page: http://www.fftw.org/

2 The box-size of the $\mathrm{L} 10$ is very small $\left(L=10 h^{-1} \mathrm{Mpc}\right.$ on a side) and hence it does not include density fluctuations larger than the box size that may affect small-scale clustering via mode coupling. To avoid this, we use only modes much smaller than the box size.
Table 1. CDM and WDM models

\begin{tabular}{lcc}
\hline \hline Model & $k_{\mathrm{fs}}\left(h \mathrm{Mpc}^{-1}\right)$ & $m_{\mathrm{WDM}}(\mathrm{keV})$ \\
\hline CDM & --- & --- \\
\hline WDM140 & 140 & 5.0 \\
\hline WDM44 & 44 & 1.9 \\
\hline WDM15 & 15 & 0.77 \\
\hline WDM4.8 & 4.8 & 0.29 \\
\hline
\end{tabular}

Note: The CDM and WDM models in our simulations. We show the free-streaming wavenumbers $k_{\mathrm{fs}}$ and WDM particle masses $m_{\mathrm{WDM}}$.

simulation (L10). The solid curves are obtained from our fitting formula based on the halofit model for a $\Lambda$ CDM model (Smith et al. 2003; Takahashi et al. 2012), but slightly modified in WDM models. Our formula is based on the numerically obtained power spectra with the maximum wavenumber $k_{\max }=300(30) h / \mathrm{Mpc}$ for the L10(L100). Details of the model fitting parameters are given in Appendix A. The simulation box-size should be much larger than the freestreaming scales in WDM models to follow gravitational evolution accurately. Thus, we do not use the simulation results for the WDM4.8 in the small-box simulation (L10), because its free-streaming scale $\left(\lambda_{\mathrm{fs}}=2 \pi / k_{\mathrm{fs}}=1.3 h^{-1} \mathrm{Mpc}\right)$ is close to the box size $\left(10 h^{-1} \mathrm{Mpc}\right)$. As shown in Fig. 3, the suppression due to free-streaming of WDM particles becomes less prominent at low redshifts even though the initial power spectra of the WDM models are exponentially suppressed at small scales $k \gtrsim k_{\mathrm{fs}}$. For example, the initial power spectrum $P(k)$ of the WDM15 is ten orders of magnitude smaller than that of the CDM at $k=300 h / \mathrm{Mpc}$, but the ratios become only $\sim 2(4)$ at low redshifts $z=0(2)$. This result exhibits power transfer from large to small scales via the mode coupling during the non-linear evolution (Bagla \& Padmanabhan 1997; White \& Croft 2000; Smith \& Markovic 2011; Viel et al. 2012). The quick regeneration of the suppressed power of WDM models and catching up with the linear and non-linear power of the CDM play an important role for estimating the lensing effects due to line-of-sight structures. The small-scale powers with wavenumbers $k>300 h / \mathrm{Mpc}$ may be systematically larger than the extrapolated values due to spurious fragmentation of filaments at the scale of grids (Wang \& White 2007). However, for fitting, we use only power spectra at scales larger than $k=300 h / \mathrm{Mpc}$ where the numerical convergence is confirmed. Therefore, such numerical noises do not affect our estimates (see Appendix A). From our simulations with $512^{3}$ and $1024^{3}$ particles, it turns out that for $k>320 h / \mathrm{Mpc}$, the error of $P(k)$ can be estimated as $\sim 10 \%$ and our fitting formula overestimates the simulation results by a factor of $1 \sim 2$ for $320<k<1000 h / \mathrm{Mpc}$. In what follows, we use $P(k)$ obtained from our fitting formula, which would yield a conservative constraint on the WDM mass. 
Table 2. Our simulation setting

\begin{tabular}{lcccccc}
\hline \hline & $L\left(h^{-1} \mathrm{Mpc}\right)$ & $N_{\mathrm{p}}^{3}$ & $k_{\mathrm{Nyq}}\left(h \mathrm{Mpc}^{-1}\right)$ & $m_{\mathrm{p}}\left(h^{-1} M_{\odot}\right)$ & $z$ & $N_{\mathrm{r}}$ \\
\hline L100 & 100 & $1024^{3}$ & 32.2 & $8.1 \times 10^{7}$ & $0,0.3,0.6,1,2,3$ & 3 \\
\hline L10 & 10 & $1024^{3}$ & 322 & $8.1 \times 10^{4}$ & $0,0.3,0.6,1,2,3$ & 5 \\
\hline
\end{tabular}

Note: Parameters in our simulations are side length of simulation box $L$, number of dark matter particles $N_{\mathrm{p}}^{3}$, Nyquist wavenumber $k_{\mathrm{Nyq}}$, particle mass $m_{\mathrm{p}}$, redshifts of the simulation outputs $z$ and number of realizations $N_{\mathrm{r}}$.

\section{LENS MODEL}

As a fiducial model of lensing galaxies, we adopt an SIE (Kormann et al. 1994), which can explain flat rotation curves. We use the fluxes of lensed images, the relative positions of lensed quadruple images and the centroid of lensing galaxies and time delay of lensed images if available. The contribution from groups, clusters, and large-scale structures at angular scales larger than the Einstein radius of the primary lens is taken into account as an external shear (ES). The parameters of the SIE('s) plus ES model are the angular scale of the critical curve or the mass scale inside the critical curve $b^{\prime}$, the apparent ellipticity $e$ of the lens and its position angle $\theta_{e}$, the strength and the direction of the ES $\left(\gamma, \theta_{\gamma}\right)$, the lens position $\left(x_{G}, y_{G}\right)$ and the source position $\left(x_{s}, y_{s}\right)$. The Hubble constant $h$ is also treated as a model parameter. The angles $\theta_{e}$ and $\theta_{\gamma}$ are measured in East of North expressed in the observer's coordinates (see Table 4).

To find a set of best-fitting parameters, we use a numerical code called GRAVLENS ${ }^{3}$ developed by Keeton in order to implement the simultaneous $\chi^{2}$ fitting of the fluxes, positions, and time delay of lensed images (if reliable data are available) and the positions of centroid of lensing galaxies. The total $\chi_{\text {tot }}^{2}$ is equal to the sum of $\chi_{\text {imag }}^{2}$ for lensed images, $\chi_{\text {flux }}^{2}$ for fluxes, $\chi_{\text {tdel }}^{2}$ for time delays, and $\chi_{\text {gal }}^{2}$ for the positions of lensing galaxies.

\section{QUADRUPLE LENS SYSTEMS}

In the following, we shortly describe six quadruple lens systems that show a large cusp relation $R_{\text {cusp }}$ or fold relation $R_{\text {fold }}{ }^{4}$. It is known that eight quadruple lens systems show apparent anomalies in the radio flux ratios (e.g., Mao \& Schneider (1998); Metcalf \& Madau (2001); Chiba (2002); Dalal \& Kochanek (2002); Metcalf et al. (2004)). However, we exclude B1555+375 and B1933+503 in our analysis since the redshifts of the lens and source of B1555+375 are not measured and the spiral lens B1933+503 has a very complex structure (Suyu et al. 2012). In our analysis, we use the observed MIR fluxes for MG0414+0534 and the radio fluxes averaged over a certain period for other five systems. It should be noted that the MIR fluxes are microlens free especially for high-redshift sources. For the astrometry, we

\footnotetext{
3 See http://redfive.rutgers.edu/ keeton/gravlens/

4 For instance, if image $\mathrm{A}$ and $\mathrm{B}$ are minima and image $\mathrm{C}$ is saddle, $R_{\text {cusp }}=\left(\mu_{A}+\mu_{C}+\mu_{B}\right) /\left(\mu_{A}+\left|\mu_{C}\right|+\mu_{B}\right)$ for the cusp lenses and $R_{\text {fold }}=\left(\mu_{A}+\mu_{C}\right) /\left(\mu_{A}+\left|\mu_{C}\right|\right)$ or $\left(\mu_{B}+\mu_{C}\right) /\left(\mu_{B}+\right.$ $\left.\left|\mu_{C}\right|\right)$ for the fold lenses.
}

use optical or NIR data in order to avoid bias due to complex structures of jets. We also use time delay for modelling $\mathrm{B} 1608+656$. We find that B2045+265 and B1608+656 with large $R_{\text {cusp }} \sim 0.5$ are no longer anomalous $\left(\chi_{\text {flux }}^{2} \leqslant 1\right.$ for each lensed image) if a companion galaxy G2 at the redshift of the primary lens G1 is taken into account. Note that our result for B2045+265 is consistent with the previous work (McKean et al. 2007). Therefore, we use four anomalous quadruple lenses B1422+231, B0128+437, MG0414+0534, and B0712+472 for constraining the mass of WDM particles. All the observed data used in our analysis are listed in table 3. In what follows, we describe the property of each lens.

\section{$5.1 \quad$ B1422+231}

The cusp-caustic lens B1422+231 consists of three bright images $\mathrm{A}, \mathrm{B}, \mathrm{C}$, and a faint image D. Images $\mathrm{A}$ and $\mathrm{C}$ are minima, and $\mathrm{B}$ and $\mathrm{D}$ are saddles. The quasar redshift $z_{S}=3.62$ is the largest in our four samples and the primary lensing galaxy is at $z_{L}=0.34$ (Kundic et al. 1997; Tonry 1998) and the measured ellipticity and the position angle of nearinfrared (near-IR) light distribution are $e=0.39 \pm 0.02$ and $\theta_{e}=-58.90 \pm 0.80\left(^{\circ}\right)$ (Sluse et al. 2012). We use the radio flux ratios (Koopmans et al. 2003) of four images at $5 \mathrm{GHz}$ averaged over a period of 8.5 months, which are consistent with the MIR counterparts (Chiba 2002). We also use the astrometry of lensed images and the centroid of the primary lensing galaxy in Sluse et al. (2012) obtained from the use of Magain-Courbin-Sohy (MCS) deconvolution algorithm applied in an iterative way (ISMCS) to near-IR Hubble Space Telescope (HST) images. The maximum total error in the positions of lensed images is 1.05 mas. Therefore, we assume an error of $\sqrt{2} \times 1.05 \sim 1.4$ mas for the angular separations of lensed images. The positions of lensed images and the centroid $\mathrm{G}$ of the primary lensing galaxy are well fitted by an SIE and an ES assuming that the error in the angular position of $\mathrm{G}$ is 0.01 arcsec. However, the flux ratios are not well fitted. We find that addition of lensing potential with lowmultipole terms $(m=3$ or $m=4)$ or changing the power index of radial profile in the mass density does not improve the fit. Chiba (2002) and Nierenberg et al. (2014) argue a presence of substructure around A. Alternatively, the possible perturber may be a halo or some other objects in the line of sight. For computing the magnification perturbation, we use only three bright images as the signal-to-noise ratio of $\mathrm{D} / \mathrm{B}$ is significantly worse than the other images. 


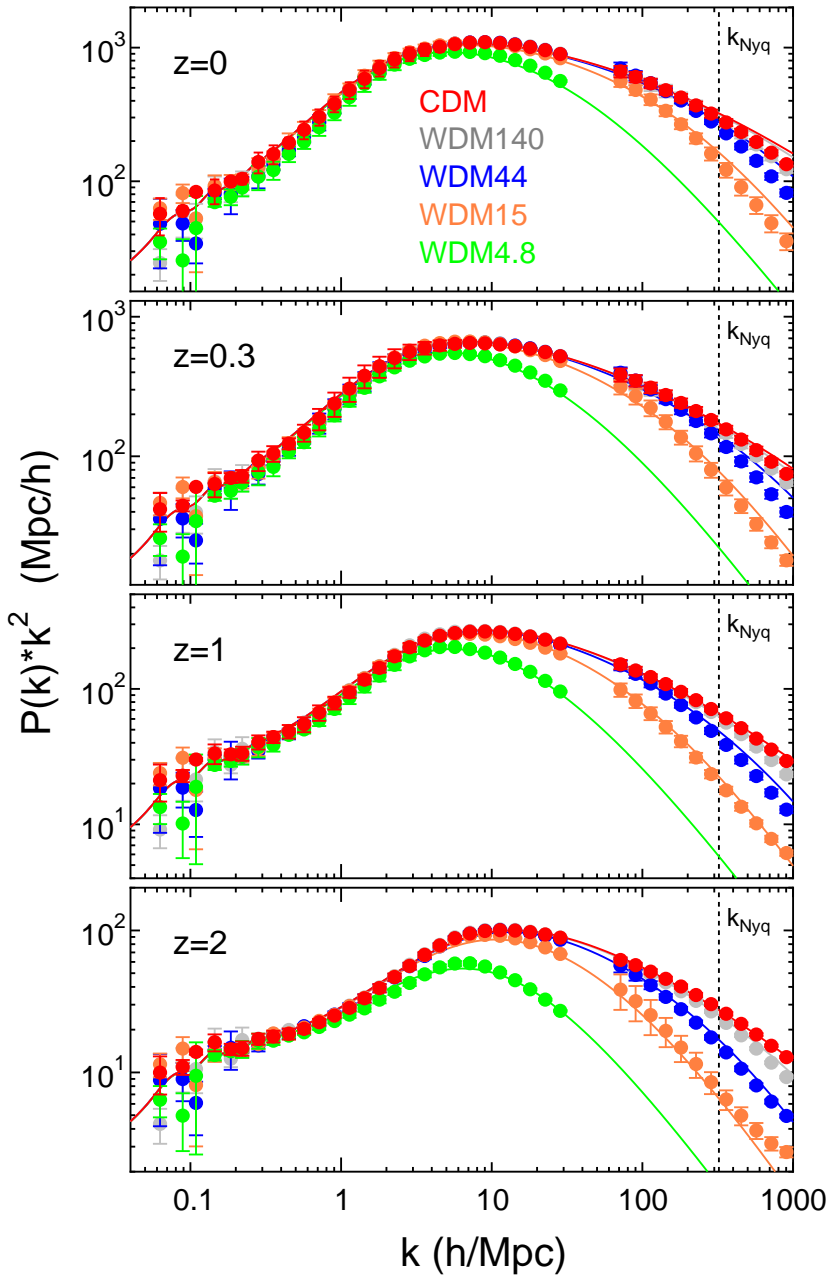

Figure 3. Non-linear matter power spectra for CDM and WDM models for various redshifts $z=0,0.3,1$ and 2 . The filled circles with the error bars are the simulation results for CDM(red), WDM140(grey), WDM44(blue), WDM15(orange) and WDM4.8(green) in Table 1. The results are taken from the large-box simulations (L100) for $k<30 h / \mathrm{Mpc}$ and the small-box simulations (L10) for $k>60 h /$ Mpc. Note that the vertical axis is $P(k) k^{2}$ (not $P(k)$ ) to show the differences among the models clearly. The vertical dotted line denotes the Nyquist wavenumber of the small-box simulations (L10). The solid curves are obtained from our fitting formula for the WDM models (see the main text and Appendix A).

\section{$5.2 \quad$ B0128+437}

The fold-caustic lens B0128+437 consists of one bright image $\mathrm{A}$, and three fainter images $\mathrm{B}, \mathrm{C}$ and $\mathrm{D}$. The images $\mathrm{A}$ and $\mathrm{C}$ are minima, and $\mathrm{B}$ and $\mathrm{D}$ are saddles. The quasar redshift is $z_{S}=3.124$ (McKean et al. 2004) and that of the primary lensing galaxy is either $z_{L}=0.645$ or 1.145 (Lagattuta et al. 2010). Combining with the previous photometric and spectroscopic data, the latter choice is favoured than the former (McKean et al. 2004; Lagattuta et al. 2010). Therefore, we assume $z_{l}=1.145$ in what follows. We use the radio flux ratios (Koopmans et al. 2003) of four images at $5 \mathrm{GHz}$ averaged over a period of 8.5 months, and the astrometry in Lagattuta et al. (2010) obtained from ground-based near-IR imaging coupled with laser guide-star adaptive optics. We also assume that the astrometric errors of each lensed image are 0.005 arcsec (Lagattuta et al. 2010). Although the positions of lensed images and the centroid of the primary lensing galaxy G can be fitted by an SIE plus an ES, the predicted flux ratios show discrepancy with the data. There might be a sub/line-of-sight halo around C.

\section{$5.3 \quad$ MG0414+0534}

The fold-caustic lens MG0414+0534 consists of two bright images $\mathrm{A} 1$ and $\mathrm{A} 2$, and two faint images $\mathrm{B}$ and $\mathrm{C}$. The images $\mathrm{A} 1$ and $\mathrm{B}$ are minima, and $\mathrm{A} 2$ and $\mathrm{C}$ are saddles. A source quasar at $z_{S}=2.64$ is lensed by an elliptical galaxy at $z_{L}=0.96$ (Hewitt et al. 1992; Lawrence et al. 1995; Tonry \& Kochanek 1999). A simple lens model, an SIE with an external shear (SIE-ES) cannot fit the image positions as well as the flux ratios. Schechter \& Moore (1993) and Ros et al. (2000) suggested that another galaxy called "X" is necessary for fitting the relative image positions. We use the MIR flux ratios A2/A1 and B/A1 measured by Minezaki et al. (2009) and MacLeod et al. (2013) since the radio fluxes might be hampered by Galactic refractive scintillation(Koopmans et al. 2003). For the astrometry, we use the data from the CASTLES (CfA-Arizona Space Telescope LEns Survey) data base of gravitational lens. Although, the positions are well fitted by an SIE and an ES plus an SIS that accounts for object $\mathrm{X}$, the flux ratios are not well fitted. A possible sub/line-of-sight halo near A2 significantly improves the fit (Minezaki et al. 2009; MacLeod et al. 2013). Note that our model is consistent with the best-fittingted macro model in MacLeod et al. (2013) without a possible subhalo G3.

\subsection{B1608+656}

The fold caustic lens B1608+656 consists of three bright images A, B, C and one faint image D. A source quasar at $z_{S}=1.394$ is lensed by two early-type galaxies G1 and G2 at $z_{L}=0.630$ (Myers \& et al. 1995; Fassnacht et al. 1996). The measured ellipticity and the position angle of near-IR light distribution are $e=0.45 \pm 0.01$ and $\theta_{e}=73.50 \pm 0.40\left(^{\circ}\right)$ for $\mathrm{G} 1$ and $e=0.55 \pm 0.01$ and $\theta_{e}=-81.10 \pm 0.20\left(^{\circ}\right)$ for G2 (Sluse et al. 2012). The lens galaxy G1 belongs to a lowmass group of eight members (Fassnacht et al. 2006). We use the astrometry of lensed images and the centroid of G1 and G2 in Sluse et al. (2012). The fluxes and time delays between these four images are based on radio-wavelength monitoring with the Very Large Array at $8.5 \mathrm{GHz}$ (Fassnacht et al. 2002). Time delay between image $A$ and $B$ is denoted as $t_{\mathrm{A}}-t_{\mathrm{B}}=\Delta t_{\mathrm{BA}}$. All the observed data are fitted well by an SIE(for G1)+ES(for environment)+SIE(for G2) model $\chi_{\text {tot }}^{2} /$ dof $=1.6$ though the best-fitting ellipticities $e(\mathrm{G} 1)=0.621, e(\mathrm{G} 2)=0.759$ are somewhat larger than the observed values in Sluse et al. (2012). The best-fitting Hubble constant $h=0.905$ is too large. However, $\sim 20 \%$ deviation could be explained by deviation from the assumed power law of mass distribution (Schneider \& Sluse 2013). 
We conclude that this system is not anomalous in the flux ratios though the cusp relation is violated as $R_{\text {cusp }} \sim 0.492$. This is due to the complex structure of the lens.

\section{$5.5 \quad \mathrm{~B} 0712+472$}

The fold-caustic lens B0712+472 consists of two bright images $\mathrm{A}$ and $\mathrm{B}$, and two fainter images $\mathrm{C}$ and $\mathrm{D}$. The source and lens redshifts are $z_{S}=1.339$ and $z_{L}=0.4060$ (Fassnacht \& Cohen 1998). We use the radio flux ratios in Koopmans et al. (2003) of four images at $5 \mathrm{GHz}$ averaged over a period of 8.5 months. For the astrometry, we use the data from CASTLES data base. The positions of lensed images and the centroid $\mathrm{G}$ of the primary lensing galaxy are well fitted by an SIE and an ES assuming that the error in the position of $\mathrm{G}$ is 0.05 arcsec. However, the flux ratios are not well fitted. For computing the magnification perturbation, we use only three bright images as the signal-to-noise ratio of $\mathrm{D} / \mathrm{A}$ is significantly worse than the other images.

\subsection{B2045+265}

The cusp-caustic lens B2045+265 consists of three bright images A, B, and C and one faint image D. The source and lens redshifts are $z_{S}=1.28$ and $z_{L}=0.8673$ (Fassnacht et al. 1999). We use the radio flux ratios (Koopmans et al. 2003 ) of four images at $5 \mathrm{GHz}$ averaged over a period of 8.5 months. For the astrometry, we use the infrared components of B2045 obtained by adaptive optics imaging at $2.2 \mu \mathrm{m}$ (McKean et al. 2007). In addition to a primary lensing galaxy $\mathrm{G} 1$ at $z_{L}$, a possible companion galaxy $\mathrm{G} 2$ may reside near G1 though the redshift has not been known(McKean et al. 2007). All the observed data are fitted extremely well by an SIE(for G1)+ES(for environment)+SIE(for G2) model giving $\chi_{\text {tot }}^{2} / \mathrm{dof}=0.03$. However, the best-fitting ellipticity of G2 seems too large $e(\mathrm{G} 2)=0.867$. Such a large value can only be expected from either an edge-on disc system or a tidally disrupted dwarf galaxy. If we do not include an SIE for G2 in the lens model, it has been known that B2045+265 exhibits strong anomaly in the flux ratios between three cusp-caustic images (Keeton et al. 2003). Since G2 resides at a position between G1 and the three cuspcaustic images A, B and C, it is natural to include the lensing effect for G2. Thus, we conclude that this system is not anomalous in the flux ratios though the cusp relation is significantly violated as $R_{\text {cusp }} \sim 0.501$.

\section{RESULTS}

As shown in Table 5, the observed magnification perturbations $\hat{\eta}$ with respect to the best-fitting lens models in Section 4 are in the range of $0.063<\hat{\eta}<0.13$. Using three lensed images $\left(N_{\text {image }}=3\right)$, we find that $\eta$ for B1422+231 is nonzero at $\sim 20 \sigma$ level, implying that the flux-ratio anomaly is most prominent. For the other three lensing systems, the significance of non-zero $\eta$ is $2 \sim 3 \sigma$.

In order to estimate the second moment of $\eta$, we have to consider a cut-off scale $k_{\max }$ that corresponds to the smallest fluctuations due to the finite size of the source. From dust reverberation, the radius of the MIR emitting region of MG0414+0534 is estimated as $\sim 2 \mathrm{pc}$ (Minezaki et al.
2009). As the magnifications of A1 and A2 images are 17, the apparent comoving size of the lensed source at the lens plane is $r_{s} \sim\left(1+z_{s}\right) \times 2 \times \sqrt{17}=30 \mathrm{pc}$. Assuming that $k_{\text {max }}=2 \pi /\left(4 r_{s}\right)$, we have $k_{\max } \sim 8 \times 10^{4} h / \mathrm{Mpc}$. For radio sources, we can estimate $k_{\max }$ from the apparent angular sizes (typically $1 \sim 3$ mas in radius) of lensed very long baseline interferometry (VLBI) images. Then we find that $3 \times 10^{3} \lesssim k_{\max } \lesssim 1 \times 10^{5}$ in units of $h / \mathrm{Mpc}$. Taking into account ambiguity in the source size, we consider two types of choices $k_{\max }=3 \times 10^{3} \mathrm{~h} / \mathrm{Mpc}$ and $10^{5} \mathrm{~h} / \mathrm{Mpc}$. The corresponding $k_{\text {cut }}$ 's for the anomalous four lenses are in the range of $530<k_{\text {cut }}<3560 h / \mathrm{Mpc}$ (Fig. 4). We find that $k_{\text {cut }}$ 's for the WDM models are equal to or less than the values for the CDM model. A larger free-streaming scale yields a larger cut-off scale $\sim 1 / k_{\text {cut }}$. Dependence of $k_{\text {cut }}$ on $k_{\max }$ is found to be very small. The expected rms $\eta$ 's for B1608+656 and B2045+265 are consistent with the null result (Table 5).

In the CDM model, we find that contribution from modes with wavelength $k>3 \times 10^{3} h / \mathrm{Mpc}$ is not negligible, especially for high-redshift sources (Table 5). This suggests that $\eta$ in CDM models is sensitive on the property of small-scale fluctuations in systems with a high-redshift source. However, for the WDM models with a large freestreaming scale, contribution from modes on small scales is very small (Fig. 5). For instance, the difference in the second moment of $\eta$ between the model with $k_{\max }=3 \times 10^{3} h / \mathrm{Mpc}$ and that with $k_{\max }=10^{5} \mathrm{~h} / \mathrm{Mpc}$ is less than $15 \%$ for $k_{\mathrm{fs}}=30 \mathrm{~h} / \mathrm{Mpc}$ (Fig. 6). The reason is as follows. As the squared amplitudes of convergence perturbation is proportional to $k^{2} P(k)$ regardless of the free-streaming scale (see Fig. 3), the modes with $k \sim k_{\text {lens }}$ contribute much to the magnification perturbation $\eta$. Moreover, the decay of power due to the free-streaming of WDM particles further reduces the contributions from small-scale modes with $k>k_{\text {lens }}$.

In order to constrain the mass of WDM particles with a free-streaming scale $k_{\mathrm{fs}}$, we use the PDFs of magnification perturbation $\eta$ for each anomalous lens system $i$. The $\mathrm{PDF}$ for system $i$ is $P\left(\eta_{i} ;\left\langle\eta_{i}^{2}\right\rangle^{1 / 2} ; \delta \eta_{i}\right)$ where $\left\langle\eta_{i}^{2}\right\rangle$ is the second moment of $\eta_{i}$, which is a function of $k_{\mathrm{fs}}$ and $\delta \eta_{i}$ is the $1-\sigma$ observational error for a lens $i$ (see Appendix B). For the null hypothesis that the observed non-vanishing $\eta_{i}$ 's are due to line-of-sight structures in the WDM model, for $N$ anomalous systems with observed magnification perturbations $\left(\hat{\eta}_{1}, \hat{\eta}_{2}, \cdots, \hat{\eta}_{N}\right)$, the $p$-value can be estimated as

$$
p\left(k_{\mathrm{fs}}\right)=\frac{\int_{S} d \boldsymbol{\eta} \prod_{i} P\left(\eta_{i} ;\left\langle\eta_{i}^{2}\right\rangle^{1 / 2} ; \delta \eta_{i}\right)}{\int_{\text {all }} d \boldsymbol{\eta} \prod_{i} P\left(\eta_{i} ;\left\langle\eta_{i}^{2}\right\rangle^{1 / 2} ; \delta \eta_{i}\right)},
$$

where $\boldsymbol{\eta}=\left(\eta_{1}, \eta_{2}, \cdots, \eta_{N}\right)$ and a domain $S$ is defined as a region where

$$
\prod_{i} P\left(\eta_{i} ;\left\langle\eta_{i}^{2}\right\rangle^{1 / 2} ; \delta \eta_{i}\right)<\prod_{i} P\left(\hat{\eta}_{i} ;\left\langle\eta_{i}^{2}\right\rangle^{1 / 2} ; \delta \eta_{i}\right)
$$

holds. For the concordant $\Lambda$ CDM model, we find that $p=$ 0.19 for $k_{\max }=3 \times 10^{3} h / \mathrm{Mpc}$ and $p=0.53$ for $k_{\max }=$ $10^{5} \mathrm{~h} / \mathrm{Mpc}$. Thus, the null hypothesis cannot be rejected. For WDM models, we find that $p<0.05$ if $k_{\mathrm{fs}}<27 h / \mathrm{Mpc}$ provided that $k_{\max }=10^{5} \mathrm{~h} / \mathrm{Mpc}$ (Fig. 7). In terms of the thermal WDM mass, the constraint can be expressed as $m_{\text {WDM }} \geqslant 1.3 \mathrm{keV}$. For the mass of sterile neutrinos, the constraint corresponds to $m_{\mathrm{s}} \gtrsim 6.3 \mathrm{keV}$. For smaller $k_{\max }$, the 
Table 3. Quadruple lens systems

\begin{tabular}{|c|c|c|c|c|c|c|}
\hline Lens system & Image(type) & Position(obs.) $\left({ }^{\prime \prime}\right)$ & Flux ratio(obs.) & $\mu($ model $)$ & Flux ratio(model) & References \\
\hline $\begin{array}{l}\mathrm{B} 1422+231 \\
z_{L}=0.34 \\
z_{S}=3.62 \\
\varepsilon=1.4 \mathrm{mas}\end{array}$ & $\begin{array}{l}\mathrm{A}(\mathrm{I}) \\
\mathrm{B}(\mathrm{II}) \\
\mathrm{C}(\mathrm{I}) \\
\mathrm{D}(\mathrm{II}) \\
\mathrm{G}\end{array}$ & $\begin{array}{c}(-0.3860 \pm 0.0004,0.3169 \pm 0.0003) \\
(0 ., 0 .) \\
(0.3360 \pm 0.0003,-0.7516 \pm 0.0005) \\
(-0.9470 \pm 0.0006,-0.8012 \pm 0.0005) \\
(-0.7321 \pm 0.0037,-0.6390 \pm 0.0054)\end{array}$ & $\begin{array}{l}\mathrm{A} / \mathrm{B}=0.9416 \pm 0.0080 \\
\mathrm{C} / \mathrm{B}=0.5188 \pm 0.0079 \\
\mathrm{D} / \mathrm{B}=0.0226 \pm 0.0057\end{array}$ & $\begin{array}{l}6.892 \\
-8.744 \\
4.327 \\
-0.334\end{array}$ & $\begin{array}{l}\mathrm{A} / \mathrm{B}=0.7882 \\
\mathrm{C} / \mathrm{B}=0.5070 \\
\mathrm{D} / \mathrm{B}=0.0368\end{array}$ & (1) (2) \\
\hline $\begin{array}{l}\mathrm{B} 0128+437 \\
z_{L}=1.145(\star) \\
z_{S}=3.124 \\
\varepsilon=4.2 \mathrm{mas}\end{array}$ & $\begin{array}{l}\mathrm{A}(\mathrm{I}) \\
\mathrm{B}(\mathrm{II}) \\
\mathrm{C}(\mathrm{I}) \\
\mathrm{D}(\mathrm{II}) \\
\mathrm{G}\end{array}$ & $\begin{array}{c}(0.000 \pm 0.002,0.0000 \pm 0.0003) \\
(-0.099 \pm 0.003,0.095 \pm 0.003) \\
(-0.521 \pm 0.004,-0.170 \pm 0.002) \\
(-0.109 \pm 0.003,-0.260 \pm 0.002) \\
(-0.217 \pm 0.01,-0.104 \pm 0.01)\end{array}$ & $\begin{array}{l}B / A=0.584 \pm 0.030 \\
C / A=0.520 \pm 0.030 \\
D / A=0.506 \pm 0.032\end{array}$ & $\begin{array}{l}5.005 \\
-2.940 \\
2.323 \\
-2.613\end{array}$ & $\begin{array}{l}\mathrm{B} / \mathrm{A}=0.587 \\
\mathrm{C} / \mathrm{A}=0.464 \\
\mathrm{D} / \mathrm{A}=0.522\end{array}$ & (1) (3) (4) \\
\hline $\begin{array}{l}\mathrm{MG} 0414+0534 \\
z_{L}=0.96 \\
z_{S}=2.639 \\
\varepsilon=4.2 \mathrm{mas}\end{array}$ & $\begin{array}{l}\text { A1(I) } \\
\text { A2(II) } \\
\text { B(I) } \\
\text { C(II) } \\
\text { G } \\
\text { X }\end{array}$ & $\begin{array}{c}(-0.600 \pm 0.003,-1.942 \pm 0.003) \\
(-0.732 \pm 0.003,-1.549 \pm 0.003) \\
(0 ., 0 .) \\
(1.342 \pm 0.003,-1.650 \pm 0.003) \\
(0.472 \pm 0.003,-1.277 \pm 0.003) \\
(0.857 \pm 0.011,0.180 \pm 0.009)\end{array}$ & $\begin{array}{l}\mathrm{A} 2 / \mathrm{A} 1=0.919 \pm 0.021 \\
\mathrm{~B} / \mathrm{A} 1=0.347 \pm 0.013 \\
\mathrm{C} / \mathrm{A} 1=0.139 \pm 0.014\end{array}$ & $\begin{array}{l}16.593 \\
-17.233 \\
5.456 \\
-2.704\end{array}$ & $\begin{array}{l}\mathrm{A} 2 / \mathrm{A} 1=1.007 \\
\mathrm{~B} / \mathrm{A} 1=0.341 \\
\mathrm{C} / \mathrm{A} 1=0.171\end{array}$ & (5) (6) (7) \\
\hline $\begin{array}{l}\mathrm{B} 1608+656 \\
z_{L}=0.630 \\
z_{S}=1.394 \\
\varepsilon=2.4 \mathrm{mas} \\
\left(\Delta t_{\mathrm{BA}}, \Delta t_{\mathrm{BC}}, \Delta t_{\mathrm{BD}}\right)(\text { days })\end{array}$ & $\begin{array}{l}\mathrm{A}(\mathrm{I}) \\
\mathrm{B}(\mathrm{I}) \\
\mathrm{C}(\mathrm{II}) \\
\mathrm{D}(\mathrm{II}) \\
\mathrm{G} 1 \\
\mathrm{G} 2\end{array}$ & $\begin{array}{c}(0 ., 0 .) \\
(-0.7464 \pm 0.0026,-1.9578 \pm 0.0026) \\
(-0.7483 \pm 0.0038,-0.4465 \pm 0.0033) \\
(1.1181 \pm 0.0025,-1.2527 \pm 0.0018) \\
(0.4561 \pm 0.0061,-1.0647 \pm 0.0037) \\
(-0.2821 \pm 0.0015,-0.9359 \pm 0.0023) \\
\left(31.5_{-1}^{+2}, 36.0 \pm 1.5,77.0_{-1}^{+2}\right)\end{array}$ & $\begin{array}{l}\mathrm{A}=3.41 \pm 0.07 \\
\mathrm{~B}=1.68 \pm 0.03 \\
\mathrm{C}=1.73 \pm 0.03 \\
\mathrm{D}=0.59 \pm 0.01\end{array}$ & $\begin{array}{l}4.904 \\
2.518 \\
-2.576 \\
-0.8692\end{array}$ & $\begin{array}{l}\mathrm{A}=3.32 \\
\mathrm{~B}=1.70 \\
\mathrm{C}=1.74 \\
\mathrm{D}=0.588\end{array}$ & (2) (8) \\
\hline $\begin{array}{c}\mathrm{B} 0712+472 \\
z_{L}=0.406 \\
z_{S}=1.339 \\
\varepsilon=6.4 \mathrm{mas}\end{array}$ & $\begin{array}{l}A(\mathrm{I}) \\
\mathrm{B}(\mathrm{II}) \\
\mathrm{C}(\mathrm{I}) \\
\mathrm{D}(\mathrm{II}) \\
\mathrm{G}\end{array}$ & $\begin{array}{c}(0.795 \pm 0.003,-0.156 \pm 0.003) \\
(0.747 \pm 0.003,-0.292 \pm 0.006) \\
(-0.013 \pm 0.004,-0.804 \pm 0.003) \\
(-0.391 \pm 0.006,-0.082 \pm 0.003) \\
(0 ., 0 .)\end{array}$ & $\begin{array}{l}\mathrm{B} / \mathrm{A}=0.843 \pm 0.061 \\
\mathrm{C} / \mathrm{A}=0.418 \pm 0.037 \\
\mathrm{D} / \mathrm{A}=0.082 \pm 0.035\end{array}$ & $\begin{array}{l}8.716 \\
-7.735 \\
3.051 \\
-0.504\end{array}$ & $\begin{array}{l}B / A=0.888 \\
C / A=0.350 \\
D / A=0.0579\end{array}$ & (1) (5) \\
\hline $\begin{array}{l}\mathrm{B} 2045+265 \\
z_{L}=0.8673 \\
z_{S}=1.28 \\
\varepsilon=1.4 \mathrm{mas}\end{array}$ & $\begin{array}{l}\mathrm{A}(\mathrm{I}) \\
\mathrm{B}(\mathrm{II}) \\
\mathrm{C}(\mathrm{I}) \\
\mathrm{D}(\mathrm{II}) \\
\text { G1 } \\
\text { G2 }\end{array}$ & $\begin{array}{c}(0.0000 \pm 0.0005,0.0000 \pm 0.0005) \\
(0.1316 \pm 0.0006,-0.2448 \pm 0.0006) \\
(0.2869 \pm 0.0005,-0.7885 \pm 0.0005) \\
(-1.6268 \pm 0.0013,-1.0064 \pm 0.0013) \\
(-1.1084 \pm 0.0011,-0.8065 \pm 0.0011) \\
(-0.4498 \pm 0.0021,-0.6425 \pm 0.0021)\end{array}$ & $\begin{array}{l}\mathrm{B} / \mathrm{A}=0.578 \pm 0.059 \\
\mathrm{C} / \mathrm{A}=0.739 \pm 0.073 \\
\mathrm{D} / \mathrm{A}=0.102 \pm 0.025\end{array}$ & $\begin{array}{l}9.515 \\
-5.531 \\
7.148 \\
-0.970\end{array}$ & $\begin{array}{l}\mathrm{B} / \mathrm{A}=0.581 \\
\mathrm{C} / \mathrm{A}=0.751 \\
\mathrm{D} / \mathrm{A}=0.102\end{array}$ & (1) (9) \\
\hline
\end{tabular}

Note: $(\star)$ : The lens redshift $z_{L}$ is obtained from a best-fitting model. References: (1) Koopmans et al. (2003) (2) Sluse et al. (2012) (3) Biggs et al. (2004) (4) Lagattuta et al. (2010) (5) CASTLES data base:http://www.cfa.harvard.edu/castles (6) Minezaki et al. (2009) (7) MacLeod et al. (2013) (8) Fassnacht et al. (2002) (9)McKean et al. (2007) Types I and II correspond to minimum and saddle, respectively. $\mu$ represents magnification.

constraint becomes more stringent. Because the power spectrum on small scales obtained from our fitting function is systematically larger than the correct values, the obtained constraint on the WDM mass is a conservative one.

\section{CONCLUSION AND DISCUSSION}

We have investigated the weak lensing effect by line-ofsight structures in a concordant CDM and WDM models based on $N$-body simulations. We have found that four quadruple lenses with source redshifts at $1 \leqslant z_{s} \leqslant 4$ out of six show anomalies in the flux ratios of lensed images assuming that the density of the primary lens is described by an SIE. The magnitudes of expected magnification perturbation due to the line-of-sight structures in the concordant $\Lambda \mathrm{CDM}$ model are consistent with the observed ones. Using four anomalous samples and extrapo- lated power spectra obtained from numerical simulations of WDM models, a constraint on the free-streaming scale of WDM particles, $k_{\mathrm{fs}} \geqslant 27 h / \mathrm{Mpc}(95 \% \mathrm{CL})$ has been obtained. For thermally produced WDMs, we have a constraint $m_{\text {WDM }} \geqslant 1.3 \mathrm{keV}(95 \% \mathrm{CL})$.

Our result for fluctuations at low-redshifts $0<z<4$ is consistent with constraints from Lyman- $\alpha$ forests at $4<z<$ 6 (Viel et al. 2013) and those from high-redshift $(4 \lesssim z \lesssim 10)$ galaxy counts (Schultz et al. 2014). Therefore, WDM models with $m_{\mathrm{WDM}}<1.3 \mathrm{keV}$ are ruled out at redshifts $0.5 \lesssim z \lesssim$ 10. Thus, WDM models as solutions for the 'missing satellite problem' are disfavoured virtually at all the redshifts.

Our calculations are based on a semi-analytic formalism that has been used for estimating magnification perturbations due to line-of-sight structures in the CDM models. In order to verify the assumed PDF form of magnification perturbation $\eta$ in WDM models (see Appendix B), we need ray- 


\section{Kaiki Taro Inoue, Ryuichi Takahashi, Tomo Takahashi, Tomoaki Ishiyama}

Table 4. best-fitting model parameters

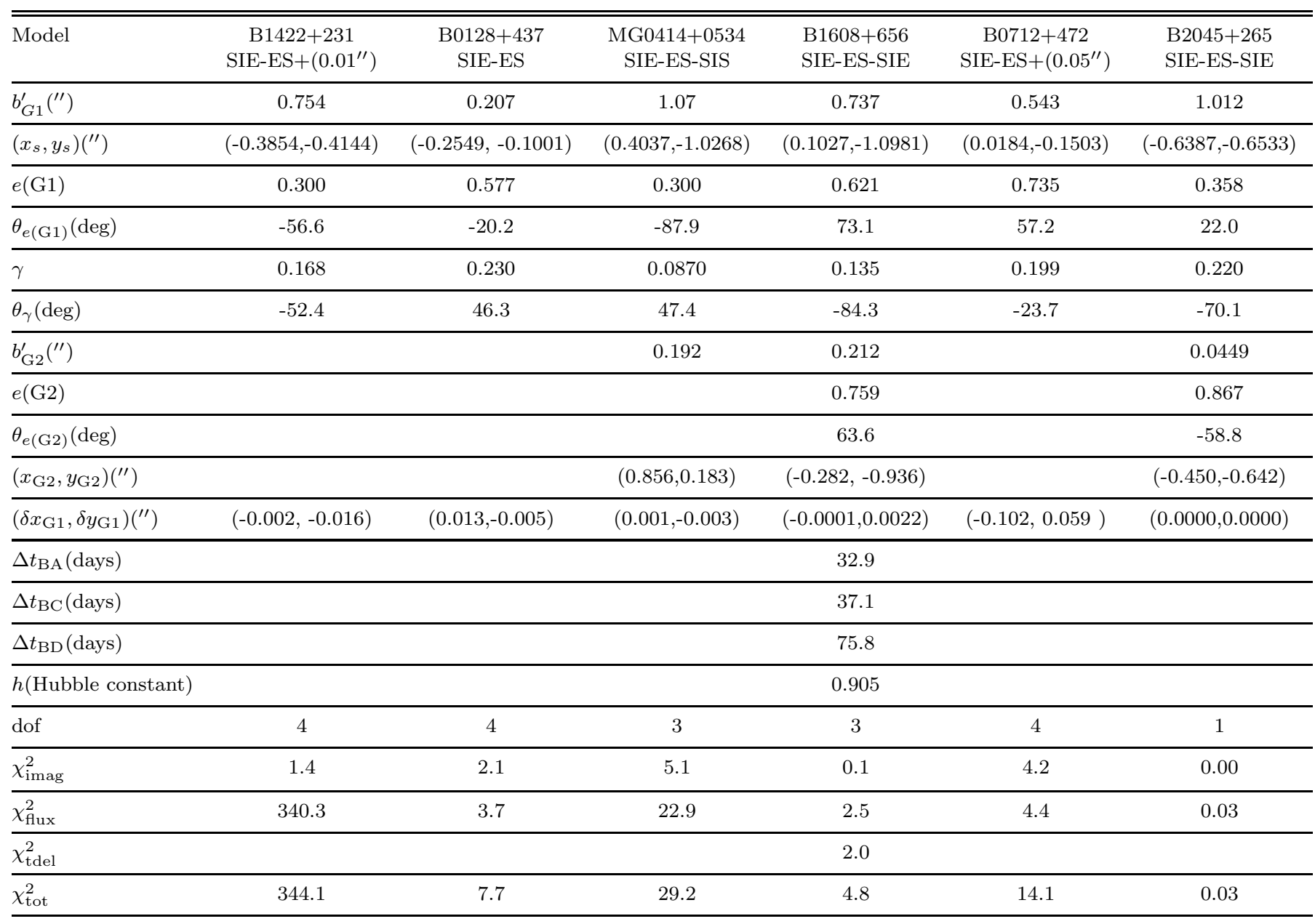

Table 5. Magnification perturbation in CDM models

\begin{tabular}{|c|c|c|c|c|c|c|c|c|}
\hline Lens system & $z_{S}$ & $z_{L}$ & $k_{\text {lens }}(h / \mathrm{Mpc})$ & $k_{\text {source }}(h / \mathrm{Mpc})$ & $N_{\text {image }}$ & $\hat{\eta}$ & $\left\langle\eta^{2}\right\rangle_{k_{\max }=3 \times 10^{3}}^{1 / 2(\mathrm{CDM})}(*)$ & $\left\langle\eta^{2}\right\rangle_{k_{\max }=10^{5}}^{1 / 2(\mathrm{CDM})}(*)$ \\
\hline B1422+231 & 3.62 & 0.34 & 412 & $\sim 7 \times 10^{4}$ & 3 & $0.098 \pm 0.005$ & $0.058(3330)$ & $0.10(3560)$ \\
\hline B0128+437 & 3.124 & 1.145 & 527 & $\sim 1 \times 10^{4}$ & 4 & $0.0632 \pm 0.025$ & $0.063(530)$ & $0.083(530)$ \\
\hline MG0414+0534 & 2.639 & 0.96 & 118 & $\sim 8 \times 10^{4}$ & 4 & $0.131 \pm 0.042$ & $0.11(1670)$ & $0.14(1720)$ \\
\hline B1608+656 & 1.394 & 0.630 & 172 & $\gtrsim 3 \times 10^{3}$ & 4 & $0.0223 \pm 0.0091$ & $0.019(2240)$ & $0.026(2290)$ \\
\hline B0712+472 & 1.339 & 0.406 & 401 & $\sim 7 \times 10^{4}$ & 3 & $0.131 \pm 0.071$ & $0.078(670)$ & $0.084(670)$ \\
\hline B2045+265 & 1.28 & 0.8673 & 134 & $\sim 8 \times 10^{4}$ & 3 & $0.075 \pm 0.050$ & No solution & $0.049(3580)$ \\
\hline
\end{tabular}

$\left(^{*}\right)$ : The values inside parentheses indicate the corresponding $k_{\text {cut }}$ in units of $h / \mathrm{Mpc}$. We estimate the $1 \sigma$ errors in $\hat{\eta}$ by assuming that errors in the observed fluxes (flux ratios) obey the Gaussian statistics with no correlation between the errors.

tracing Monte Carlo simulations where the lens parameter fitting is done with the presence of line-of-sight structures, which will be our future work.

In our simulations, we did not take into account nonluminous subhaloes in lensing galaxies. In CDM models, it has been shown that the surface mass density of subhaloes in lensing galaxies are not enough for explaining the observed flux-ratio anomalies (Amara et al. 2006; Maccio \& Miranda 2006; Chen 2009; Xu et al. 2009, 2010; Chen et al. 2011). As the number density of subhaloes with sizes that are comparable to or less than the free-streaming scale $\sim 1 / k_{\mathrm{fs}}$ is significantly reduced, the role of dark subhaloes in lensing galaxies would be minor. However, we may need to check the lensing effects of subhaloes in WDM models as well. 


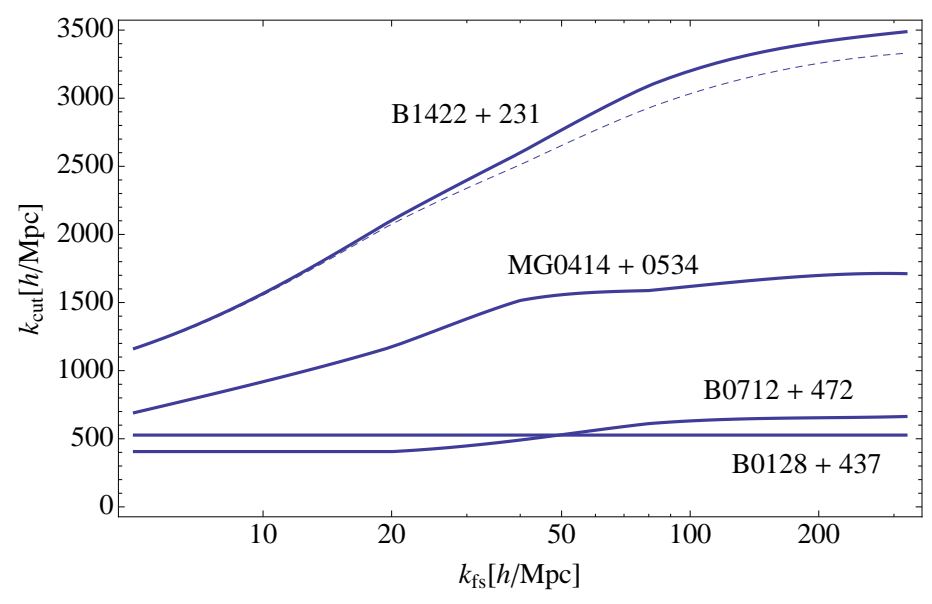

Figure 4. Dependence of $k_{\text {cut }}$ on the free streaming scale. $k_{\text {cut }}$ is plotted as a function of $k_{\mathrm{fs}}$ for $k_{\max }=10^{5} \mathrm{~h} / \mathrm{Mpc}$ (full curve) and $k_{\max }=3 \times 10^{3} h / \mathrm{Mpc}$ (dashed curve). Except for B1422+231, the dashed curves almost coincide with the full curves.

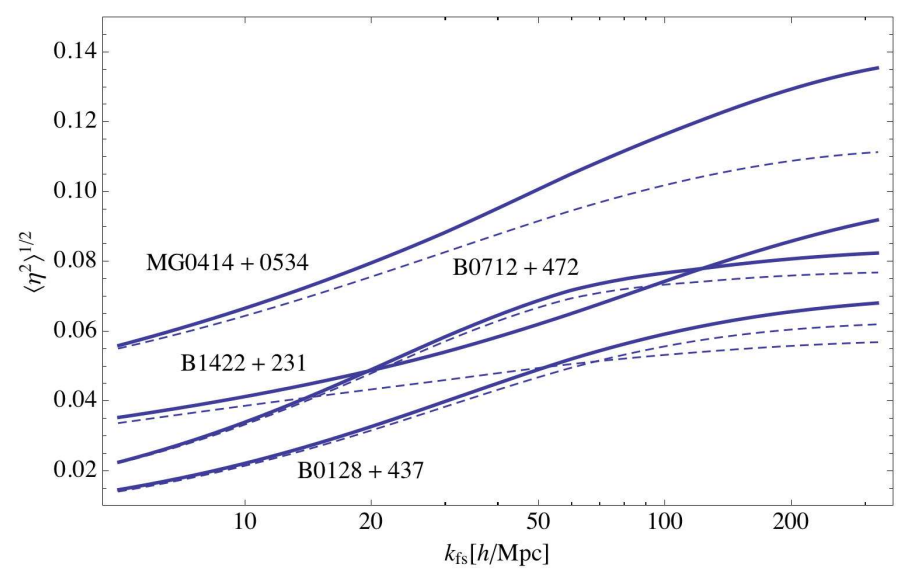

Figure 5. Suppression of magnification perturbation due to freestreaming. The square-root of second moment $\left\langle\eta^{2}\right\rangle^{1 / 2}$ is plotted as a function of $k_{\mathrm{fs}}$ for $k_{\max }=10^{5} h / \mathrm{Mpc}\left(\right.$ full curve) and $k_{\max }=$ $3 \times 10^{3} \mathrm{~h} / \mathrm{Mpc}$ (dashed curve).

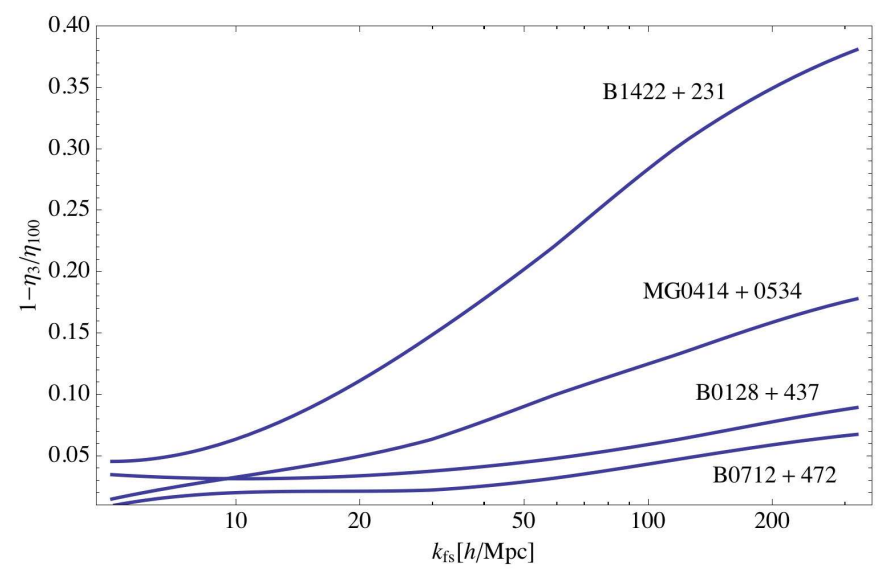

Figure 6. Effect of small-scale fluctuations. $\eta_{3}$ and $\eta_{100}$ correspond to $\left\langle\eta^{2}\right\rangle^{1 / 2}$ for $k_{\max }=3 \times 10^{3} h / \mathrm{Mpc}$ and $k_{\max }=$ $10^{5} \mathrm{~h} / \mathrm{Mpc}$, respectively.

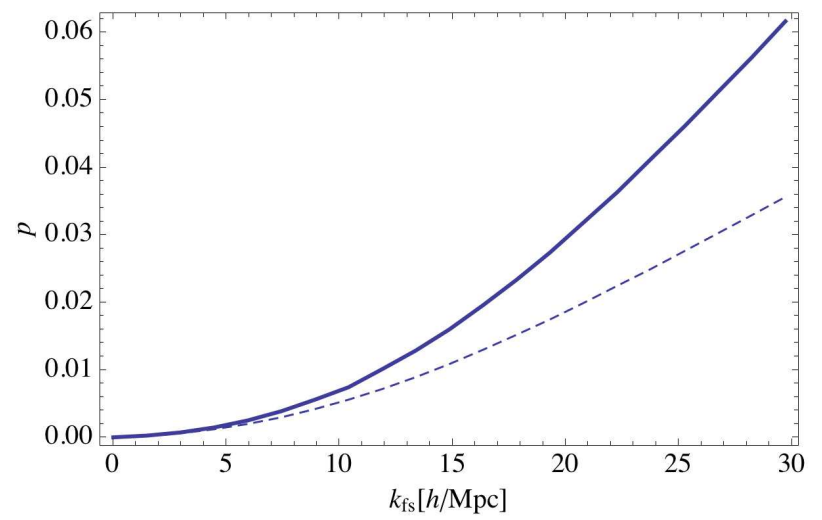

Figure 7. Plots of $p$-value as a function of $k_{\mathrm{fs}}$ for $k_{\max }=$ $10^{5} h / \mathrm{Mpc}$ (full curve) and $k_{\max }=3 \times 10^{3} h / \mathrm{Mpc}($ dashed curve).

We expect that baryonic feedback effects on the lineof-sight structures are limited to the central region of minihaloes. Therefore, the weak lensing effects that are relevant to the property of outskirts of minihaloes may not change so much. Although, the power spectra from simulations with baryons may significantly differ, those calculated from fluctuations obtained by masking the central regions of haloes would be less affected. In order to verify it, however, it is very important to incorporate baryonic effects in our lensing simulations, which will also be our future work.

Another important issue is the ambiguity in the macro lens model. Although we have assumed that the density of the macro lens can be described by an SIE plus an ES, the actual lens may have a more complex structure, such as anisotropy in the velocity dispersion and deviation from power laws, etc. Therefore, caution has to be made that the constraints on the WDM mass could be weakened if the applied macroscopic mass models based on SIEs with a constant ES are not proper ones (Xu et al. 2014).

However, in the near future, we will obtain a larger sample of lens and more precise information about the macro lens model [e.g., the Atacama Large Millimeter/submillimeter Array (ALMA) and Thirty Meter Telescope], which have a potential for breaking degeneracy in the lens model.

If intervening perturbers are massive enough $(\gtrsim$ $\left.10^{10} M_{\odot}\right)$, we may directly detect the presence and the redshift of perturbers from the extended-source effects (Inoue \& Chiba 2005b,a). In order to do so, observation of anomalous quadruple lenses by ALMA is important. Emission from neutral hydrogen (HI) may be another clue for detecting the line-of-sight structures. Measuring correlation between flux-ratio anomaly and HI emission may be a new test for confirming the presence of line-of-sight structures.

\section{ACKNOWLEDGEMENTS}

We thank the anonymous referees for his/her valuable comments. This work is supported in part by JSPS Grant-inAid for Scientific Research (B) (No. 25287062) "Probing the origin of primordial minihaloes via gravitational lensing phenomena". The work of TT is partially supported by the Grant-in-Aid for Scientific Research from the Min- 


\section{Kaiki Taro Inoue, Ryuichi Takahashi, Tomo Takahashi, Tomoaki Ishiyama}

istry of Education, Science, Sports, and Culture, Japan, No. 23740195. TI is financially supported by MEXT HPCI Strategic Program and MEXT/JSPS KAKENHI Grant Number 24740115. Numerical computations were carried out on Cray XT4 at Center for Computational Astrophysics, CfCA, at National Astronomical Observatory of Japan and the $\mathrm{K}$ computer at the RIKEN Advanced Institute for Computational Science (proposal numbers hp120286 and hp130026).

\section{APPENDIX A: FITTING FORMULA FOR NON-LINEAR MATTER POWER SPECTRA IN WDM MODELS}

In this appendix, we present our fitting formula for the matter power spectra in WDM models. Our formula is based on the halofit model (Smith et al. 2003; Takahashi et al. 2012), but slightly modified for WDM models.

To find the best fitting parameters in the theoretical model, we use the standard chi-square fitting, which is defined as

$$
\chi^{2}=\sum_{i} \sum_{z=0}^{3} \sum_{k=k_{\min }}^{k_{\max }} \frac{\left[P_{i, \text { model }}(k, z)-P_{i, \operatorname{sim}}(k, z)\right]^{2}}{P_{i, \operatorname{sim}}(k, z)^{2}},
$$

where $P_{i, \text { model }}$ is the power in the theoretical model, $P_{i \text {,sim }}$ 's are those in simulation results, and $i$ denotes the CDM model $(i=0)$ and the four WDM models $(i=1-4)$ in Table 1. The $\chi^{2}$ is summed over redshifts $z=0,0.3,0.6,1,2$ and 3 . We use the wavenumber $k$ larger than $2 h / \operatorname{Mpc}\left(=k_{\min }\right)$ where the Gaussian error of $P(k)$ is less than $1 \%$. The maximum wavenumber is $k_{\max }=300(30) h / \mathrm{Mpc}$ for the L10(L100) so that the measured power spectrum is much larger (10 times larger) than the shot noise.

First, we fit the simulation results of the CDM model. The fitting parameters for the CDM model are the same as in Takahashi et al. (2012), except for the following three parameters:

$$
\begin{aligned}
& \log _{10} a_{\mathrm{n}}= 0.9221+2.0595 n_{\mathrm{eff}}+2.4447 n_{\mathrm{eff}}^{2}+1.2625 n_{\mathrm{eff}}^{3} \\
&+0.2874 n_{\mathrm{eff}}^{4}-0.7601 C \\
& \log _{10} c_{\mathrm{n}}= 0.4747+2.1542 n_{\mathrm{eff}}+0.8582 n_{\mathrm{eff}}^{2}+0.8329 C \\
& \gamma_{\mathrm{n}}=0.2247-0.2287 n_{\mathrm{eff}}+0.9726 C-0.0533 \ln \left(\frac{k}{h / \mathrm{Mpc}}\right) .
\end{aligned}
$$

The ratios of the power spectra of the WDM to that of the $\mathrm{CDM}$ are fitted as,

$$
\frac{P_{\mathrm{wdm}}(k, z)}{P_{\mathrm{cdm}}(k, z)}=\frac{1}{\left(1+k / k_{\mathrm{d}}\right)^{0.7441}},
$$

with

$$
k_{\mathrm{d}}\left(k_{\mathrm{fs}}, z\right)=2.206 h \mathrm{Mpc}^{-1}\left(\frac{k_{\mathrm{fs}}}{h / \mathrm{Mpc}}\right)^{1.703} D(z)^{1.583},
$$

where $k_{\mathrm{fs}}$ is the free-streaming wavenumber and $D(z)$ is the linear growth factor at $z$, which is normalized as $D(z=0)=$ 1. Equation (A4) can be rewritten in terms of the WDM particle mass $m_{\mathrm{WDM}}$ as

$$
k_{\mathrm{d}}\left(k_{\mathrm{fs}}, z\right)=388.8 h \mathrm{Mpc}^{-1}\left(\frac{m_{\mathrm{WDM}}}{\mathrm{keV}}\right)^{2.027} D(z)^{1.583} .
$$

The RHS of equation (A3) corresponds to a damping factor. Note that the parameters $n_{\text {eff }}$ and $C$ in equation (A2) are evaluated in the CDM model even when computing $P_{\mathrm{wdm}}(k, z)$. Using our fitting formula, the simulation results can be reproduced within a relative error of $19 \%$. The rms deviation between the theoretical model and the simulation results is about $4.5 \%$.

Fig.A1 shows the ratio of the WDM power spectrum $P_{\mathrm{WDM}}(k, z)$ to the CDM power spectrum $P_{\mathrm{CDM}}(k, z)$ at redshifts $z=0,0.3,1$ and 2 . The solid curves represent the decay of power spectra described by our damping factor in equation (A3), which reproduces our simulation results very well. For comparison, the predicted power spectra based on the previous fitting formula in Viel et al. (2012) are plotted as dotted curves. As shown in Fig.A1, the predicted powers based on the previous fitting formula are too large at scales $k \sim 100 h / \mathrm{Mpc}$. The discrepancy becomes more prominent at smaller scales $k \gtrsim 100 h / \mathrm{Mpc}$. This is probably due to the fact that the Nyquist wavenumber $k_{\mathrm{Nyq}}=60 h / \mathrm{Mpc}$ (see fig.7 in Viel et al. (2012)) in their simulations is smaller than ours $k_{\mathrm{Nyq}}=322 h / \mathrm{Mpc}$.

\section{APPENDIX B: FUNCTIONAL FORM OF PDF}

In this appendix, we provide the PDFs of $\eta$. We assume that the PDFs are approximated by the log-normal function as

$$
P(\eta)=N \exp \left[-\frac{1}{2 \sigma^{2}}\left\{\ln \left(1+\frac{\eta}{\eta_{0}}\right)-\ln \mu\right\}^{2}\right] \frac{1}{\eta+\eta_{0}}
$$

where $N$ is a normalization constant, $\eta_{0}$ describes a dispersion scale of $\eta$, and $\sigma$ and $\mu$ are constants. We assume that $\eta_{0}$ depends only on the second moment $\left\langle\eta^{2}\right\rangle$ and that $\sigma$ and $\mu$ do not depend on $\left\langle\eta^{2}\right\rangle$. Using ray-tracing simulations for a concordant $\Lambda \mathrm{CDM}$ model, we find that the best-fitting parameters are (Takahashi \& Inoue 2014)

$$
\mu=4.10, \sigma^{2}=0.279, \eta_{0}=0.228\left\langle\eta^{2}\right\rangle^{1 / 2} .
$$

As the formula (B1) does not depend on the grid size $r_{\text {grid }}$ of the simulation (see Fig.9 in Takahashi \& Inoue (2014)), we assume that the formula (B1) is also applicable to WDM models where small-scale fluctuations are suppressed due to free-streaming ${ }^{5}$. We find that the suppression in the power due to the finite grid size $\sim 4.8 \mathrm{kpc} / h$ is comparable to the one by free-streaming for $k_{\mathrm{fs}} \sim 20 \mathrm{~h} / \mathrm{Mpc}$.

In real setting, we need to take into account errors in observation as well. If the variance of observational error is $\delta \eta^{2}$, and the mean value is vanishing, we should change $\eta_{0}$ as

$$
\eta_{0}=0.228\left(\left\langle\eta^{2}\right\rangle+\delta \eta^{2}\right)^{1 / 2}
$$

\footnotetext{
${ }^{5}$ Even in CDM models, the slopes of density profiles of haloes on scales equal to or less than the free streaming scale are different from those on larger scales (e.g. Ishiyama et al. (2010); Ishiyama (2014)). However, the difference is striking only in the very inner regions (within $10 \%$ of the virial radius) of haloes. Since the contribution to the PDF of $\eta$ due to the weak lensing effects mainly comes from the outer regions (>virial radius), it would be reasonable to assume that the statistics of $\eta$ in WDM models are the same as those in CDM models.
} 


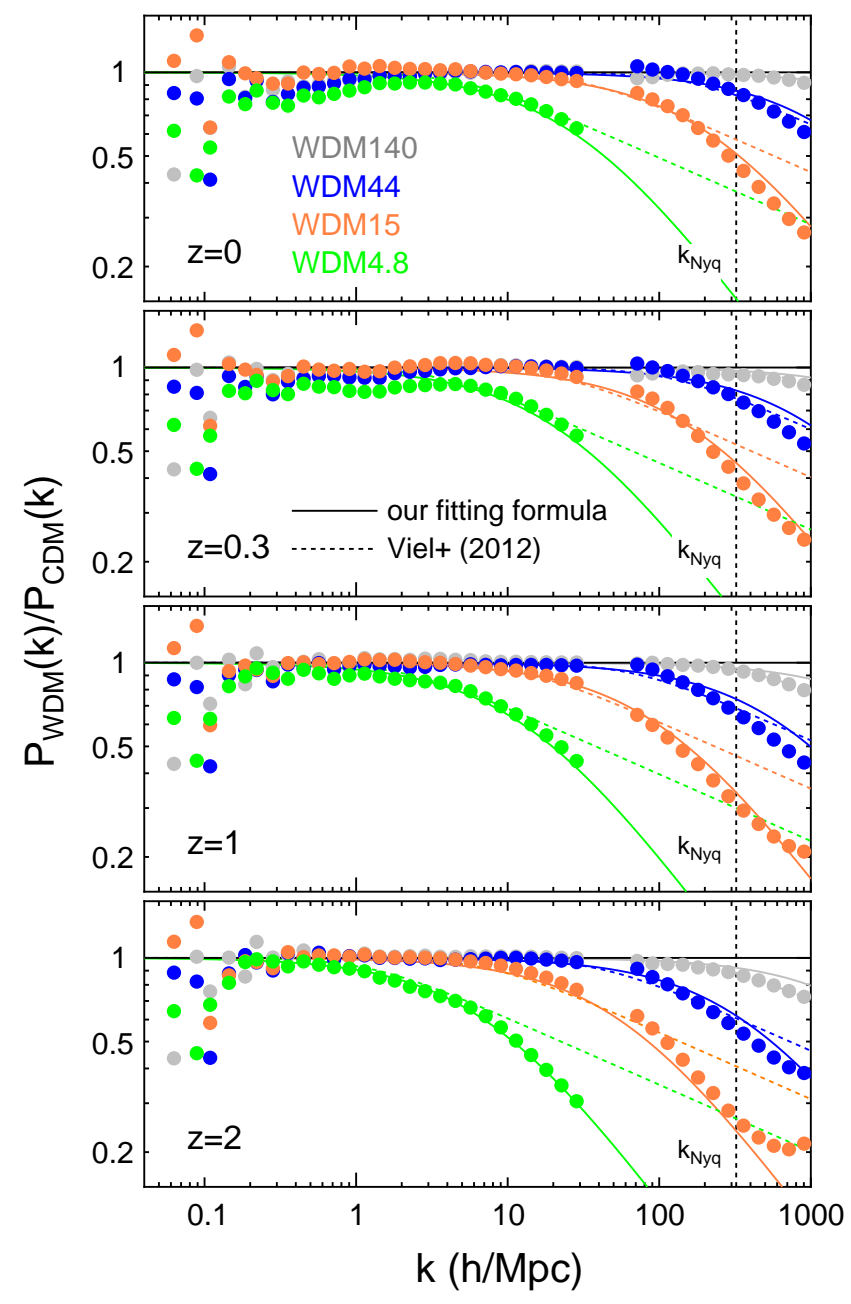

Figure A1. Same as Fig.3, but the ratio of the WDM power spectra $P_{\mathrm{WDM}}(k)$ to the $\mathrm{CDM} P_{\mathrm{CDM}}(k)$. The filled circles are the simulation results for WDM140(grey), WDM44(blue), WDM15(orange) and WDM4.8(green). The solid curves correspond to our fitting formula in equation (A3), while the dotted curves to the previous one in Viel et al. (2012).

\section{REFERENCES}

Amara A., Metcalf R. B., Cox T. J., Ostriker J. P., 2006, Monthly Notices of the Royal Astronomical Society, 367, 1367

Angulo R. E., Hahn O., Abel T., 2013, Monthly Notices of Royal Astronomical Society, 434, 3337

Bagla J. S., Padmanabhan T., 1997, Monthly Notices of Royal Astronomical Society, 286, 1023

Biggs A. D., Browne I. W. A., Jackson N. J., York T., Norbury M. A., McKean J. P., Phillips P. M., 2004, Monthly Notices of Royal Astronomical Society, 350, 949

Bode P., Ostriker J. P., Turok N., 2001, The Astrophysical Journal, 556, 93

Boehm C., Mathis H., Devriendt J., Silk J., 2005, Monthly Notices of Royal Astronomical Society, 360, 282
Boyarsky A., Lesgourgues J., Ruchayskiy O., Viel M., 2009, Journal of Cosmology and Astroparticle Physics, 5, 12

Chen J., 2009, Astronomy \& Astrophysics, 498, 49

Chen J., Koushiappas S. M., Zentner A. R., 2011, Astrophysical Journal, 741

Chen J., Kravtsov A. V., Keeton C. R., 2003, Astrophysical Journal, 592, 24

Chiba M., 2002, The Astrophysical Journal, 565, 17

Chiba M., Minezaki T., Kashikawa N., Kataza H., Inoue K. T., 2005, Astrophysical Journal, 627, 53

Colombi S., Dodelson S., Widrow L. M., 1996, Astrophysical Journal, 458, 1

Crocce M., Pueblas S., Scoccimarro R., 2006, Monthly Notices of the Royal Astronomical Society, 373, 369

Dalal N., Kochanek C. S., 2002, The Astrophysical Journal, 572,25

Dodelson S., Widrow L. M., 1994, Physical Review Letters, 72,17

Fassnacht C. D., Blandford R. D., Cohen J. G., Matthews K., Pearson T. J., Readhead A. C. S., Womble D. S., Myers S. T., Browne I. W. A., Jackson N. J., Marlow D. R., Wilkinson P. N., Koopmans L. V. E., de Bruyn A. G., Schilizzi R. T., Bremer M., Miley G., 1999, Astrophysical Journal, 117, 658

Fassnacht C. D., Cohen J. G., 1998, Astrophysical Journal, 115,377

Fassnacht C. D., Gal R. R., Lubin L. M., McKean J. P., Squires G. K., Readhead A. C. S., 2006, The Astrophysical Journal, 642, 30

Fassnacht C. D., Womble D. S., Neugebauer G., Browne I. W. A., Readhead A. C. S., Matthews K., Pearson T. J., 1996, The Astrophysical Journal, 460, L103

Fassnacht C. D., Xanthopoulos E., Koopmans L. V. E., Rusin D., 2002, The Astrophysical Journal, 581, 823

Hewitt J. N., Turner E. L., Lawrence C. R., Schneider D. P., Brody J. P., 1992, Astrophysical Journal, 104, 968

Hockney R., Eastwood J., 1988, Computer simulation using particles. Taylor \& Francis, New York

Inoue K. T., Chiba M., 2005a, Astrophysical Journal, 634, 77

Inoue K. T., Chiba M., 2005b, Astrophysical Journal, 633, 23

Inoue K. T., Takahashi R., 2012, Monthly Notices of Royal Astronomical Society, 426, 2978

Ishiyama T., 2014, Astrophysical Journal, 788, 27

Ishiyama T., Fukushige T., Makino J., 2009, Publications of the Astronomical Society of Japan, 61, 1319

Ishiyama T., Makino J., Ebisuzaki T., 2010, Astrophysical Journal, 723, L195

Ishiyama T., Nitadori K., Makino J., 2012, Proc. Int. Conf. High Performance Computing, Networking, Storage and Analysis, SC '12 (Los Alamitos, CA: IEEE Computer Society Press), 5, (arXiv:1211.4406)

Jenkins A., Frenk C. S., Pearce F. R., Thomas P. A., Colberg J. M., White S. D. M., Couchman H. M. P., Peacock J. A., Efstathiou G., Nelson A. H., 1998, Astrophysical Journal, 499, 20

Keeton C. R., Gaudi B. S., Petters A. O., 2003, Astrophysical Journal, 598, 138

Kolb E. W., Turner M. S., 1990, The early universe.

Koopmans L. V. E., Biggs A., Blandford R. D., Browne I. W. A., Jackson N. J., Mao S., Wilkinson P. N., de Bruyn 


\section{Kaiki Taro Inoue, Ryuichi Takahashi, Tomo Takahashi, Tomoaki Ishiyama}

A. G., Wambsganss J., 2003, The Astrophysical Journal, 595,712

Kormann R., Schneider P., Bartelmann M., 1994, Astronomy and Astrophysics, 284, 285

Kundic T., Hogg D. W., Blandford R. D., Cohen J. G., Lubin L. M., Larkin J. E., 1997, Astronomical Journal, 114,2276

Lagattuta D. J., Auger M. W., Fassnacht C. D., 2010, The Astrophysical Journal, 716, L185

Lawrence C. R., Elston R., Januzzi B. T., Turner E. L., 1995, Astronomical Journal, 110, 2570

Lewis A., Challinor A., Lasenby A., 2000, Astrophysical Journal, 538, 473

Maccio A. V., Miranda M., 2006, Monthly Notices of the Royal Astronomical Society, 368, 599

McKean J. P., Koopmans L. V. E., Flack C. E., Fassnacht C. D., Thompson D., Matthews K., Blandford R. D., Readhead A. C. S., Soifer B. T., 2007, Monthly Notices of the Royal Astronomical Society, 378, 109

MacLeod C. L., Jones R., Agol E., Kochanek C. S., 2013, The Astrophysical Journal, 773, 35

Mao S., Schneider P., 1998, Monthly Notices of the Royal Astronomical Society, 295, 587

McKean J. P., Koopmans L. V. E., Browne I. W. A., Fassnacht C. D., Blandford R. D., Lubin L. M., Readhead A. C. S., 2004, Monthly Notices of Royal Astronomical Society, 350, 167

Metcalf R. B., 2005, The Astrophysical Journal, 629, 673

Metcalf R. B., Madau P., 2001, The Astrophysical Journal, 563,9

Metcalf R. B., Moustakas L. A., Bunker A. J., Parry I. R., 2004, Astrophysical Journal, 607, 43

Minezaki T., Chiba M., Kashikawa N., Inoue K. T., Kataza H., 2009, Astrophysical Journal, 697, 610

Miranda M., Maccio A. V., 2007, Monthly Notices of the Royal Astronomical Society, 382, 1225

More A., McKean J. P., More S., Porcas R. W., Koopmans L. V. E., Garrett M. A., 2009, Monthly Notices of the Royal Astronomical Society, 394, 174

Myers S. T., et al. 1995, The Astrophysical Journal, 447, L5

Nierenberg A. M., Treu T., Wright S. A., Fassnacht C. D., Auger M. W., 2014, ArXiv e-prints

Nishimichi T., Shirata A., Taruya A., Yahata K., Saito S., Suto Y., Takahashi R., Yoshida N., Matsubara T., Sugiyama N., Kayo I., Jing Y. P., Yoshikawa K., 2009, Publications of the Astronomical Society of Japan, 61, 321

Planck Collaboration Ade P. A. R., Aghanim N., Alves M. I. R., Armitage-Caplan C., Arnaud M., Ashdown M., Atrio-Barandela F., Aumont J., Aussel H., et al. 2014, Astronomy and astrophysics, 571, A1

Ros E., Guirado J. C., Marcaide J. M., Perez-Torres M. A., Falco E. E., Munoz J. A., Alberdi A., Lara L., 2000, Astronomy and Astrophysics, 362, 845

Schechter P. L., Moore C. B., 1993, Astronomical Journal, 105, 1

Schneider A., Smith R. E., Macciò A. V., Moore B., 2012,

Monthly Notices of Royal Astronomical Society, 424, 684 Schneider P., Sluse D., 2013, Astronomy and astrophysics, 559, A37

Schultz C., Oñorbe J., Abazajian K. N., Bullock J. S., 2014,
Monthly Notices of Royal Astronomical Society, 442, 1597 Seljak U., Makarov A., McDonald P., Trac H., 2006, Physical Review Letters, 97, 191303

Sluse D., Chantry V., Magain P., Courbin F., Meylan G., 2012, Astronomy and Astrophysics, 538, A99

Smith R. E., Markovic K., 2011, Physical Review D, 84, 063507

Smith R. E., Peacock J. A., Jenkins A., White S. D. M., Frenk C. S., Pearce F. R., Thomas P. A., Efstathiou G., Couchman H. M. P., 2003, Monthly Notices of the Royal Astronomical Society, 341, 1311

Springel V., 2005, Monthly Notices of the Royal Astronomical Society, 364, 1105

Springel V., Yoshida N., White S. D. M., 2001, New Astronomy, 6, 79

Sugai H., Kawai A., Shimono A., Hattori T., Kosugi G., Kashikawa N., Inoue K. T., Chiba M., 2007, Astrophysical Journal, 660, 1016

Suyu S. H., Hensel S. W., McKean J. P., Fassnacht C. D., Treu T., Halkola A., Norbury M., Jackson N., Schneider P., Thompson D., Auger M. W., Koopmans L. V. E., Matthews K., 2012, The Astrophysical Journal, 750, 10

Takahashi R., Inoue K. T., 2014, Monthly Notices of Royal Astronomical Society, 440, 870

Takahashi R., Sato M., Nishimichi T., Taruya A., Oguri M., 2012, Astrophysical Journal, 761, 152

Tonry J. L., 1998, Astronomical Journal, 115, 1

Tonry J. L., Kochanek C. S., 1999, Astronomical Journal, 117,2034

Viel M., Becker G. D., Bolton J. S., Haehnelt M. G., 2013, Physical Review D, 88, 043502

Viel M., Lesgourgues J., Haehnelt M. G., Matarrese S., Riotto A., 2005, Physical Review D, 71, 063534

Viel M., Markovic K., Baldi M., Weller J., 2012, Monthly Notices of Royal Astronomical Society, 421, 50

Wang J., White S. D. M., 2007, Monthly Notices of Royal Astronomical Society, 380, 93

White M., Croft R. A. C., 2000, Astrophysical Journal, 539, 497

Xu D., Mao S., Wang J., Springel V., Gao L., White S., Frenk C., Jenkins A., Li G., Navarro J., 2009, Monthly Notices of the Royal Astronomical Society, 398, 1235

Xu D., Sluse D., Gao L., Wang J., Frenk C., Mao S., Schneider P., Springel V., 2014, ArXiv e-prints

Xu D. D., Mao S., Cooper A. P., Gao L., Frenk C. S., Angulo R. E., Helly J., 2012, Monthly Notices of the Royal Astronomical Society, 421, 2553

Xu D. D., Mao S. D., Cooper A. P., Wang J., Gao L. A., Frenk C. S., Springel V., 2010, Monthly Notices of the Royal Astronomical Society, 408, 1721 